\title{
Closed-Loop Equilibrium Reinsurance-Investment Strategy with Insider Information and Default Risk
}

\author{
Peng Yang ${ }^{1,2}$ \\ ${ }^{1}$ School of Science, Xijing University, Xi'an 710123, China \\ ${ }^{2}$ School of Mathematics and Statistics, Xi'an Jiaotong University, Xi'an 710049, China \\ Correspondence should be addressed to Peng Yang; yangpeng511@163.com
}

Received 27 September 2020; Revised 1 December 2020; Accepted 28 December 2020; Published 19 January 2021

Academic Editor: Francesco Clementi

Copyright $(0) 2021$ Peng Yang. This is an open access article distributed under the Creative Commons Attribution License, which permits unrestricted use, distribution, and reproduction in any medium, provided the original work is properly cited.

This paper studies the closed-loop equilibrium reinsurance-investment problem with insider information and default risk. The financial market consists of one risky asset, one defaultable bond, and one risk-free asset. The surplus process is governed by a jump-diffusion process. Two kinds of dependencies between the insurance market and the financial market are considered. In addition, the insurer has some extra claims information available from the beginning of the trading interval. The objective of the insurer is to choose a time-consistent reinsurance-investment strategy so as to maximize the expected terminal wealth while minimizing the variance of the terminal wealth. Since this problem is time-inconsistent, using closed-loop control approach from the perspective of game theory, we establish the extended Hamilton-Jacobi-Bellman (HJB) equations for the postdefault case and the predefault case, respectively. Closed-form solutions for the closed-loop equilibrium reinsurance-investment strategy and the corresponding value function are obtained. Finally, we provide a series of numerical examples to illustrate the effects of insider information and other some important model parameters on the closed-loop equilibrium reinsurance and investment strategies. The result analyses reveal some interesting phenomena and provide useful guidances for reinsurance and investment in reality.

\section{Introduction}

Mean-variance portfolio selection is a classic and an important problem in mathematical economics, which is pioneered by Markowitz [1] and has long been recognized as the milestone of modern portfolio theory. The dynamic extended of the mean-variance portfolio problem, especially in continuous time, has been widely studied. For example, Zhou and $\mathrm{Li}$ [2] investigated a continuous-time meanvariance portfolio problem in a stochastic linear quadratic framework, Bauerle [3] studied the optimal proportional reinsurance/new business problem under the mean-variance criterion, and Liang et al. [4] considered the mean-variance reinsurance and investment problem in a jump-diffusion financial market with common shock dependence.

The above mean-variance portfolio selection problem is time-inconsistent, and there are two reasons for this. First, the variance operator does not satisfy the smoothing property. Second, the optimal strategies are derived under the assumption that the investors precommit themselves not to deviate from the strategies chosen at the initial time. However, time consistency of strategy is an important and a rational requirement in many practice situations. For example, a few years ago, the Chinese government decided to get rid of poverty by 2020 . Naturally, they hope to get rid of poverty at 2020. A decision-maker sitting at time $t$ would consider that, starting from time $t+\varepsilon, \varepsilon>0$, he will follow the strategy that is optimal sitting at time $t+\varepsilon$. Namely, the optimal strategy derived at time $t$ should agree with the optimal strategy derived at time $t+\varepsilon$.

To obtain the time-consistent strategy, two kinds of equilibrium strategies are developed: closed-loop equilibrium strategy and open-loop equilibrium strategy. The primitive idea of the closed-loop equilibrium strategy can be traced back to Strotz [5]. To the best of our knowledge, Ekeland and Lazrak [6] first investigated the closed-loop equilibrium strategy for the continuous-time framework and proposed a precise definition of the game theoretic 
equilibrium strategy. Following the definition of the game theoretic equilibrium strategy, Björk and Murgoci [7] proposed the closed-loop equilibrium strategy in detail under the discrete-time setting and continuous-time setting. They handled a general class of time-inconsistent problems under Markovian framework by viewed them as intrapersonal games and looked for subgame perfect Nash equilibrium strategies. In the continuous-time case, they formally derived the extended HJB equations in conjunction with the verification theorem by using the conclusions of the discrete-time case. Recently, some scholars have applied the closed-loop control framework to study the time-consistent reinsurance-investment problem. Lin and Qian [8] considered the time-consistent reinsurance-investment problem under the constant elasticity of variance (CEV) model. Zhang and Liang [9] studied the time-consistent reinsurance-investment problem with common shock dependence and state dependent risk aversion parameter. $\mathrm{Hu}$ and Wang [10] investigated the time-consistent reinsurance-investment problem for two competitive insurers. Yang et al. [11] studied the time-consistent combining quota-share and excess of loss reinsurance and investment problem for a mean-variance insurer. Yang et al. [12] investigated the time-consistent reinsurance-investment problem under a new interaction mechanism and a general investment framework. In contrast, inspired by the idea of stochastic maximum principle, $\mathrm{Hu}$ et al. [13] studied a class of timeinconsistent stochastic linear quadratic problems in a nonMarkovian setting, introduced open-loop equilibrium controls, derived general sufficient conditions by solving a system of backward stochastic differential equations. The same authors continued to study the uniqueness of openloop equilibrium controls in $\mathrm{Hu}$ et al. [14]. Recently, some scholars have applied the open-loop control framework to study the reinsurance-investment or portfolio problems. Sun and Guo [15] studied a time-inconsistent linear quadratic control problem with stochastic coefficients and random jumps and then studied its application in mean-variance portfolio selection problem. Yan and Wong [16] investigated the mean-variance portfolio problem in incomplete markets with stochastic volatility. The same authors continued to study the reinsurance-investment problem under the general stochastic volatility model in Yan and Wong [17]. Wang [18] investigated the uniqueness issue of open-loop equilibrium investment strategies of dynamic mean-variance portfolio selection problem with stochastic coefficients.

The closed-loop equilibrium strategy is associated with the extended of dynamic programming approach, which is obtained by solving the extended HJB equation. The closedloop equilibrium strategy is often used to solve complex control problems; however, its defect is that it is difficult to guarantee the uniqueness of the strategy. The open-loop equilibrium strategy is associated with the maximum principle approach, which is obtained by solving the backward stochastic differential equation. The open-loop equilibrium strategy is easy to study the uniqueness of the strategy; however, its defect is that it cannot reflect the feedback of the system; hence, it lacks accuracy. Nowadays, the closed-loop equilibrium strategy and the open-loop equilibrium strategy are still two main methods to obtain the time-consistent strategy.

Asymmetric information plays an important role in decision-making as there are agents that have access to different levels of information. An important class of asymmetric information is the inside information, according to which the investor has some information about the future. Baltas et al. [19] first introduced the concept of inside information to the insurance market, and their objective was to maximize the expected utility of the terminal wealth. Cao et al. [20] also considered the inside information in insurance market, and they obtained the closed-form solutions for the optimal time-consistent investment and reinsurance strategies. In the studies of Baltas et al. [19] and Cao et al. [20], the insurance model was modulated by a diffusion process, and Xiong et al. [21] studied the inside information in insurance market where the insurance model was modeled by a jump-diffusion process.

Motivated by the work of Baltas et al. [19], Xiong et al. [21], and Cao et al. [20], we investigate the closed-loop equilibrium investment and reinsurance strategies with inside information. Different from Baltas et al. [19], Xiong et al. [21], and Cao et al. [20], both the insurance model and the price process of the risky asset are modeled by the jumpdiffusion process in this paper. In the studies of Baltas et al. [19], Xiong et al. [21], and Cao et al. [20], they did not consider the dependence between the insurance market and the financial market; however, they are often dependence in many circumstances. For example, the global financial crisis in 2008 and the COVID-19 will inevitably have a common influence on the two markets. Hence, we consider the dependence between the two markets in this paper, specifically, the dependence between the insurance market and the financial market consisting of the following two parts. First, the aggregate claim process and the aggregate price jump process are modeled by a common Poisson process. Second, the Brownian motion in the surplus process and the Brownian motion in the price process of the risky asset are correlated. In addition, we consider the default risk, i.e., the insurer invests in a financial market consisting of one riskfree asset, one risky asset, and one defaultable bond. The motivation to introduce the default risk is that in today's financial markets, the default of one company usually has a strong influence on other companies. Recently, the reinsurance-investment problem with default risk has become an important research topic. For example, Zhao et al. [22] considered the time-consistent reinsurance-investment problem with defaultable security for Black-Scholes financial market, Zhang and Qiao [23] investigated the same problem as that in the work of Zhao et al. [22], where the price process of the risky asset follows the CEV model, and Zhu et al. [24] considered the non-zero-sum stochastic differential reinsurance and investment games with default risk.

At present, scholars consider the insider information and the default risk separately. To the best of our knowledge, this is the first paper studies the insider information and the default risk simultaneously. This is the main contribution of this paper. Using a game theoretic approach, we establish the 
extended HJB equations for the postdefault case and the predefault case, respectively. Closed-form solutions for the closed-loop equilibrium reinsurance-investment strategy and the corresponding value function are obtained. Finally, we provide a series of numerical examples to illustrate the effects of insider information and other some important model parameters on the closed-loop equilibrium reinsurance and investment strategies. The result analyses reveal some interesting phenomena and provide useful guidances for reinsurance and investment in reality.

The rest of the paper is organized as follows. The models and the assumptions are described in Section 2. In Section 3, the time-consistent mean-variance problem with inside information and default risk under the game theoretic framework is formulated; meanwhile, the extended HJB equations and verification theorem are provided. In Section 4 , the closed-form solutions for the predefault case and the postdefault case are derived, respectively. Section 5 illustrates our theoretical results through numerical examples. The final section summarizes this paper.

\section{Model Formulation}

In this section, we present a framework for a continuoustime insurance-financial model with inside information and default risk. The insurance model is described by the jumpdiffusion process. The insurer has some extra claims information, i.e., the inside information about the future realization of the uncertain claims. The reinsuranceinvestment horizon is $[0, T]$ with $T>0$, and the financial market consists of three financial assets, namely, one riskfree asset (bond), one risky asset (stock), and one defaultable bond. We suppose that the insurance market and the financial market are frictionless, i.e., the securities can be traded continuously over time and there are no transaction costs or taxes in trading.

Let $W_{1}(t)$ and $W_{2}(t)$ be two standard Brownian motions, which are correlated with correlation coefficient being $\rho \in[-1,1]$. We provide three mutually independent Poisson processes $N_{1}(t), N_{2}(t)$, and $N(t)$, which with intensities being $\lambda_{1}>0, \lambda_{2}>0$, and $\lambda>0$, respectively. We denote

$$
\begin{aligned}
K_{1}(t) & =N_{1}(t)+N(t), \\
K_{2}(t) & =N_{2}(t)+N(t), \\
Y(t) & =\sum_{i=1}^{K_{1}(t)} Y_{i}, \\
U(t) & =\sum_{i=1}^{K_{2}(t)} U_{i} .
\end{aligned}
$$

Here, $\left\{Y_{i}, i=1,2, \ldots\right\}$ and $\left\{U_{i}, i=1,2, \ldots\right\}$ are sequences of random variables, respectively. Let $\left(\Omega, \mathscr{F}_{t}, \mathbb{F}, P\right)$ be a complete probability space with filtration $\mathscr{F}_{t}$; here, $P$ represents the probability measure, $\mathbb{F}=\left\{\mathscr{F}_{t}, 0 \leq t \leq T\right\}$, and $\mathscr{F}_{t}$ is a natural filtration generated by $W_{1}(t), W_{2}(t)$, $Y(t)$, and $U(t)$, i.e., $\mathscr{F}_{t}=\sigma\left(W_{1}(t), W_{2}(t), Y(t), U(t)\right)$.
2.1. The Surplus Process. The surplus process without reinsurance is described as follows:

$$
X(t)=x_{0}+c t+\sigma_{1} W_{1}(t)-Y(t) .
$$

Here, $x_{0} \geq 0$ denotes the initial capital, $c>0$ is the rate of premium per unit time, $\sigma_{1}>0$, and $\sigma_{1} W_{1}(t)$ stands for the uncertainty associated with the insurer's claims. $Y(t)=\sum_{i=1}^{K_{1}(t)} Y_{i}, Y_{i}$ is the size of the $i$ th claim, and $\left\{Y_{i}, i=1,2, \ldots\right\}$ forms a sequence of independent and identically distributed (i.i.d.) nonnegative random variables. The common distribution function is $F_{Y}(y)$, the common density function is $f_{Y}(y)$, and $Y_{i}$ has finite mean $\mu_{11}=$ $E\left(Y_{i}\right)$ and second moment $\mu_{12}=E\left(Y_{i}^{2}\right)$. Thus, the compound Poisson process $Y(t)$ represents the cumulative amount of claims up to time $t . K_{1}(t)=N_{1}(t)+N(t)$ is a counting process, representing the number of claims up to time $t$. Since $N_{1}(t)$ and $N(t)$ are mutually independent Poisson processes with intensities being $\lambda_{1}$ and $\lambda, K_{1}(t)$ is a Poisson process with intensity being $\lambda_{1}+\lambda$.

The insurer can control his claim risk by purchasing proportional reinsurance or acquiring new business, such as acting as a reinsurer, see Bauerle [3] for details. The proportional reinsurance level is associated with the value $1-a(t)$, where $a(t)$ represents the retention level of the insurer. Here, $0 \leq a(t) \leq 1$ corresponds to a proportional reinsurance and $a(t)>1$ corresponds to a new business requirement. The reinsurance premium rate is calculated according to the expected value principle; then, the reinsurance premium rate at time $t$ is $(1-a(t)) c_{1}$, and $c_{1}$ is the reinsurer's premium rate. With the proportional reinsurance or new business being incorporated, the surplus process $X_{t}^{a}$ of the insurer becomes

$$
d X_{t}^{a}=\left[c-(1-a(t)) c_{1}\right] d t+a(t) \sigma_{1} d W_{1}(t)-a(t) d Y(t) .
$$

2.2. The Insider Information. Inside information, as an important class of asymmetric information, plays an important role in insurance practice. Modeling the behavior of an insider is a very important and challenging problem with the most common approach adopted in the literature to be the enlargement of filtration technique. That is to say, the insider, i.e., the insurer has access to a filtration $\mathscr{G}_{t}$, which is strictly larger than the filtration $\mathscr{F}_{t}$ generated by the surplus process and the price process of the risky asset. In this paper, we assume that the insurer has some additional information about the future realizations of the uncertain claims. More precisely, the insurer has some insider information stored in the filtration $\left\{\mathscr{G}_{t}\right\}_{0 \leq t \leq T}$, where $\left\{\mathscr{G}_{t}\right\}_{0 \leq t \leq T}=\left\{\mathscr{F}_{t}\right\}_{0 \leq t \leq T} \vee \sigma$ $\left(W_{1}\left(T_{0}\right)\right)$, and $T_{0}>T$. It is clear that $\left\{\mathscr{F}_{t}\right\}_{0 \leq t \leq T}$ C $\left\{\mathscr{G}_{t}\right\}_{0 \leq t \leq T}$. Please notice that the Brownian motion $W_{1}(t)$ is not a martingale under the filtration $\mathscr{G}_{t}$. To obtain a martingale under the filtration $\mathscr{G}_{t}$, we provide the following theorem. 
Theorem 1. The process $W_{1}(t)$ is a semimartingale with respect to $\mathbb{G}=\left\{\mathscr{G}_{t}, 0 \leq t \leq T\right\}$, and its semimartingale decomposition is

$$
W_{1}(t)=\int_{0}^{t} \delta(s) \mathrm{d} s+\bar{W}_{1}(t), \quad 0 \leq t \leq T_{0}
$$

where $\delta(t)=\left(W_{1}\left(T_{0}\right)-W_{1}(t) / T_{0}-t\right)$, and $\bar{W}_{1}(t)$ is a $(\mathbb{G}, P)$ Brownian motion; i.e., $\bar{W}_{1}(t)$ is a martingale under the filtration $\mathscr{G}_{t}$.

Proof. Since the proof is similar to that in the work of Baltas et al. [19] and Protter [25], the details are omitted.

Remark 1. Theorem 1 plays a crucial role in this paper, which is considered as the basic reference about the effect of inside information on the insurance market. In Theorem 1, $\delta(t)$ is called the information drift induced by $W_{1}\left(T_{0}\right)$, which is the drift to eliminate in order to preserve the martingale property of $W_{1}(t) ; \int_{0}^{t} \delta(s) \mathrm{d} s$ is $\mathscr{G}_{t}$ adapted, continuous, and of finite variation. Since the correlation coefficient between Brownian motions $W_{1}(t)$ and $W_{2}(t)$ is $\rho$, the correlation coefficient between Brownian motions $\bar{W}_{1}(t)$ and $W_{2}(t)$ also is $\rho$.

Lemma 1. From Proposition 3.1 in the study of Baltas et al. [19], we have

$$
d W_{1}(t)=\left(\frac{W_{1}\left(T_{0}\right)}{T_{0}}-\int_{0}^{t} \frac{1}{T_{0}-s} d \bar{W}_{1}(s)\right) \mathrm{d} t+d \bar{W}_{1}(t)
$$

Let

$$
\begin{aligned}
\delta_{0} & =\lim _{t \longrightarrow 0} \delta(t)=\frac{W_{1}\left(T_{0}\right)}{T_{0}}, \\
M(t) & =\int_{0}^{t} \frac{1}{T_{0}-s} d \bar{W}_{1}(s),
\end{aligned}
$$

then we have

$$
d W_{1}(t)=\left(\delta_{0}-M(t)\right) d t+d \bar{W}_{1}(t) .
$$

Let $\bar{X}_{t}^{a}$ be the surplus process of the insurer taking into account the inside information. Substituting (7) into (3), we obtain

$$
\begin{aligned}
d \bar{X}_{t}^{a}= & {\left[c-(1-a(t)) c_{1}+a(t) \sigma_{1}\left(\delta_{0}-M(t)\right)\right] d t } \\
& +a(t) \sigma_{1} d \bar{W}_{1}(t)-a(t) d Y(t)
\end{aligned}
$$

2.3. The Financial Market. To increase wealth, the insurer invests in the financial market consisting of one risk-free asset, one risky asset, and one defaultable bond. The price process of the risk-free asset under the probability measure $P$ follows

$$
\begin{gathered}
d B(t)=r B(t) d t, \\
B(0)=b_{0}>0,
\end{gathered}
$$

where $r>0$ denotes the risk-free return rate.

The price process $S(t)$ of the risky asset under probability measure $P$ is described by the following jump-diffusion process:

$$
\begin{aligned}
d S(t) & =S(t-)\left[\mu d t+\sigma_{2} d W_{2}(t)+d U(t)\right], \\
S(0) & =s_{0} .
\end{aligned}
$$

Here, $\mu \geq r$ and $\sigma_{2}>0$ are the expected return rate and the instantaneous volatility of the risky asset, respectively. $U_{i}$ is the size of the $i$ th jump, and $U(t)=\sum_{i=1}^{K_{2}(t)} U_{i},\left\{U_{i}, i=\right.$ $1,2, \ldots\}$ is a sequence of i.i.d. nonnegative random variables. The common distribution function is $F_{U}(u)$, the common density function is $f_{U}(u)$, and $U_{i}$ with finite mean $\mu_{21}=$ $E\left(U_{i}\right)$ and second moment $\mu_{22}=E\left(U_{i}^{2}\right) . K_{2}(t)=N_{2}(t)+$ $N(t)$ is a counting process, representing the number of jumps up to time $t$. Since $N_{2}(t)$ and $N(t)$ are mutually independent Poisson processes with intensities being $\lambda_{2}$ and $\lambda, K_{2}(t)$ is a Poisson process with intensity being $\lambda_{2}+\lambda$. Thus, the compound Poisson process $U(t)$ represents the cumulative amount of jumps up to time $t$.

We consider a defaultable zero-coupon bond with unit face value and maturity $T_{1}$ and that the default time is $\tau$. In most caes, $\tau$ represents the first jump time of a Poisson process with a constant intensity $h^{P}>0$ under the probability measure $P$. Let $\zeta(\zeta \in[0,1])$ denote the loss rate of the bond. At time of default, we adopt the recovery of market value and the default recovery rate is $1-\zeta$.

Define a default indicator process $Z_{t}=\mathbb{q}_{\tau \leq t}, \mathscr{H}_{t}=\sigma$ $\left(Z_{u}, 0 \leq u \leq t\right)$ is the natural filtration generated by $\left\{Z_{t}\right\}_{t \geq 0}$, and $\mathscr{D}_{t}=\mathscr{G}_{t} \vee \mathscr{H}_{t}$ with $t \geq 0$ is the augmented filtration. Furthermore, the default process $\left\{\bar{M}_{t}^{P}\right\}$ is defined by

$$
\bar{M}_{t}^{P}=Z_{t}-\int_{0}^{t}\left(1-Z_{u-}\right) h^{P} \mathrm{~d} u .
$$

Notice that $\left\{\bar{M}_{t}^{P}\right\}$ is a martingale under the probability measure $P$.

Unlike the price process of the risk-free asset and the risky asset which are given under the real-world probability measure $P$ directly, the price process of the defaultable bond is firstly defined under the risk neutral probability measure $Q$ and then will be transformed into the price process under the probability measure $P$. Using arbitrage-free argument, at time of default, the bond's holder can receive

$$
(1-\zeta) E^{Q}\left[e^{-r\left(T_{1}-\tau\right)}\right]
$$

By the Girsanovs theorem (Lemma 2 in the work of Bielecki and Jang [26]), we know that under the probability measure $Q$, the arrival intensity of default is given by $h^{Q}=\left(h^{P} / \Delta\right)$, where $(1 / \Delta) \geq 1$ is called the jump risk premium.

To obtain the wealth process under the probability measure $P$, we now need to deduce the price of the defaultable bond under the probability measure $P$ from that under the probability measure $Q$. The following lemma, which is adapted from Lemma 3 in the work of Bielecki and Jang [26], gives the dynamics of the defaultable corporate bond under the probability measure $P$. 
Lemma 2. For positive interest rate $r$ of the risk-free asset, the P-dynamics of the defaultable bond is represented as follows:

$$
\begin{aligned}
d P\left(t, T_{1}\right)= & P\left(t-, T_{1}\right)\left[r d t+\left(1-Z_{t}\right) \delta(1-\Delta) d t\right. \\
& \left.-\left(1-Z_{t-}\right) \zeta d \bar{M}_{t}^{P}\right],
\end{aligned}
$$

where $\delta=\zeta h^{Q}$ represents the risk neutral credit spread.

Remark 2. From the above discuss, we find that the dependence between the insurance market and the financial market consists of two aspects. On the one hand, the uncertainty associated with the insurer's surplus process and the risky asset's price process is correlated; i.e., the correlation coefficient between Brownian motions $\bar{W}_{1}(t)$ and
$W_{2}(t)$ is $\rho$. On the other hand, the aggregate claim process and the aggregate price jump process is modulated by a common Poisson process $N(t)$, i.e., the number of claims is $K_{1}(t)=N_{1}(t)+N(t)$ and the number of price jumps is $K_{2}(t)=N_{2}(t)+N(t)$.

2.4. The Wealth Process. Let $\pi_{1}(t)$ and $\pi_{2}(t)$ denote the dollar amounts that the insurer invests in the risky asset and the defaultable bond at time $t$, and the rest of his wealth is then invested in the risk-free asset. The trading strategy is denoted by $\pi(t)=\left(a(t), \pi_{1}(t), \pi_{2}(t)\right)$ such that $T<T_{0} \vee T_{1}$. Under the strategy $\pi(t)$, the insurer's wealth process $\left\{X_{t}^{\pi}\right\}_{t \in[0, T]}$ satisfies the following equation:

$$
\begin{aligned}
d X_{t}^{\pi}= & \pi_{1}(t) \frac{d S(t)}{S(t)}+\pi_{2}(t) \frac{d P\left(t, T_{1}\right)}{P\left(t, T_{1}\right)}+\left(X_{t}^{\pi}-\pi_{1}(t)-\pi_{2}(t)\right) \frac{d B(t)}{B(t)}+d \bar{X}_{t}^{a} \\
= & {\left[c-(1-a(t)) c_{1}+a(t) \sigma_{1}\left(\delta_{0}-M(t)\right)+r X_{t}^{\pi}+\pi_{1}(t)(\mu-r)+\pi_{2}(t)\left(1-Z_{t}\right)(1-\Delta) \delta\right] \mathrm{d} t } \\
& +a(t) \sigma_{1} d \bar{W}_{1}(t)+\pi_{1}(t) \sigma_{2}(t) d W_{2}(t)-a(t) d Y(t) \\
& +\pi_{1}(t) d U(t)-\pi_{2}\left(1-Z_{t}\right) \zeta d \bar{M}_{t}^{P},
\end{aligned}
$$

with initial capital $X_{0}^{\pi}=x_{0}$ and

$$
d M(t)=\frac{1}{T_{0}-t} d \bar{W}_{1}(t)
$$

Definition 1. For any fixed $t \in[0, T]$, a strategy $\pi(t)=\left(a(t), \pi_{1}(t), \pi_{2}(t)\right)$ is said to be admissible if

(i) $\pi(t)$ is $\mathscr{D}_{t}$-progressively measurable

(ii) $a(t) \geq 0, E\left[\int_{0}^{T} a^{2}(t)\right]<\infty$, and $E\left[\int_{0}^{T}\left(\pi_{1}^{2}(t)+\pi_{2}^{2}\right.\right.$ $(t)) \mathrm{d} t]<\infty$, where the filtration used for the expectation operator is $\mathscr{D}_{t}$

(iii) stochastic differential equation (14) has a unique strong solution

We denote the set of all admissible strategies on the time interval $[0, T]$ by $\Pi$.

\section{Time-Consistent Mean-Variance Problem with Inside Information}

In this section, we construct a time-consistent mean-variance strategy selection problem under inside information and default risk. For convenience, let $\mathcal{O}:=[0, T] \times R \times$ $R^{+} \times\{0,1\}$. For any $(t, x, m, z) \in \mathcal{O}$, the insurer's objective is to maximize the expected terminal wealth while minimizing the variance of the terminal wealth. That is,

$$
\sup _{\pi \in \Pi} J(t, x, m, z, \pi)=\sup _{\pi \in \Pi}\left\{E_{t, x, m, z}\left[X_{T}^{\pi}\right]-\frac{\gamma}{2} \operatorname{Var}_{t, x, m, z}\left[X_{T}^{\pi}\right]\right\}
$$

where $\quad E_{t, x, m, z}(\cdot)=E\left[\cdot \mid X_{t}^{\pi}=x, M(t)=m, Z(t)=z\right]$, $\operatorname{Var}_{t, x, m, z}(\cdot)=\operatorname{Var}\left[\cdot \mid X_{t}^{\pi}=x, M(t)=m, Z(t)=z\right]$, the filtration used for the expectation and the variance operators is $\mathscr{D}_{t}$, and $\gamma$ is a prespecified risk aversion parameter of the insurer. Problem (16) is time-inconsistent since there is a nonlinear function of the terminal wealth in the variance term and for detailed proof, readers can refer to Czichowsky [27]. In practice, the consistency of strategy is a basic requirement of rational decision-making under many situations, and today's preference may be different from tomorrow's preference. Due to the lack of the iterated expectation property, the traditional dynamic programming approach does not apply directly. Therefore, inspired by Bjö rk and Murgoci [7], we will solve problem (16) in a game theoretic approach and derive the closed-loop equilibrium strategy which is time-consistent. More specifically, our problem can be regarded as a noncooperative game, with one player for each time $t$, where player $t$ can be seen as the future incarnation of the insurer at time $t$.

Remark 3. Problem (16) can be equivalently expressed as the following problem:

$$
\sup _{\pi \in \Pi} \widetilde{J}(t, x, m, z, \pi)=\sup _{\pi \in \Pi}\left\{E_{t, x, m, z}\left[F\left(X_{T}^{\pi}\right)\right]+G\left(E_{t, x, m, z}\left(X_{T}^{\pi}\right)\right)\right\} \text {, }
$$

where $F(y)=y-(1 / 2) \gamma y^{2}$ and $G(y)=(1 / 2) \gamma y^{2}$. This problem can be viewed as a dynamic mean-variance problem, since the objective of the insurer updates as state $(t, x, m, z)$ changes. 
Remark 4. Problem (16) (or problem (17)) is a special case of the following problem:

$$
\begin{aligned}
\sup _{\pi \in \Pi} \widehat{J}(t, x, m, z, \pi) & =\sup _{\pi \in \Pi}\left\{E_{t, x, m, z}\left[X_{T}^{\pi}\right]-\frac{\gamma(x)}{2} \operatorname{Var}_{t, x, m, z}\left[X_{T}^{\pi}\right]\right\} \\
& =\sup _{\pi \in \Pi}\left\{E_{t, x, m, z}\left[F\left(x, X_{T}^{\pi}\right)\right]+G\left(x, E_{t, x, m, z}\left(X_{T}^{\pi}\right)\right)\right\},
\end{aligned}
$$

where $F(x, y)=y-(\gamma(x) / 2) y^{2}, G(x, y)=(\gamma(x) / 2) y^{2}$, and $\gamma(x)$ is a state dependent risk aversion function. Obviously, if set $\gamma(x)=\gamma$, we can obtain problem (16) (or problem (17)) from problem (18). In this paper, we only study problem (16), and problem (18) will be studied in the future.

Now, we provide the definition of the equilibrium strategy for problem (16).
Definition 2. For any fixed chosen initial state $(t, x, m$, $z) \in \mathcal{O}$, consider an admissible strategy $\pi^{*}(t, x, m, z)$. Choose four fixed real numbers $\widetilde{a} \in R, \widetilde{b} \in R, \widetilde{c} \in R^{+}$, and $\varepsilon>0$, and define the following strategy:

$$
\pi^{\varepsilon}(s, \tilde{x}, \widetilde{m}, \widetilde{z})= \begin{cases}(\widetilde{a}, \widetilde{b}, \widetilde{c}), & \text { for }(s, \widetilde{x}, \widetilde{m}, \widetilde{z}) \in[t, t+\varepsilon] \times R \times R^{+} \times\{0,1\}, \\ \pi^{*}(s, \widetilde{x}, \widetilde{m}, \widetilde{z}), & \text { for }(s, \widetilde{x}, \widetilde{m}, \widetilde{z}) \in(t+\varepsilon, T] \times R \times R^{+} \times\{0,1\}\end{cases}
$$

If

$$
\liminf _{\varepsilon \longrightarrow 0} \frac{J\left(t, x, m, z, \pi^{*}\right)-J\left(t, x, m, z, \pi^{\varepsilon}\right)}{\varepsilon} \geq 0,
$$

for all $(\widetilde{a}, t \widetilde{b} n, q \widetilde{c}) \in R \times R \times R^{+}$and $(t, x, m, z) \in \mathcal{O}$, then $\pi^{*}(t, x, m, z)$ is called an equilibrium strategy. The value function $V(t, x, m, z)$ is defined by

$$
V(t, x, m, z)=J\left(t, x,, m, z, \pi^{*}\right) .
$$

Let $C^{1,2,2}(\mathcal{O})$ denote the space of $\phi(t, x, m, z)$ such that $\phi(t, x, m, z)$ is differential in $t$ and twice differential in $x$ and $m$. For any $\phi(t, x, m, z) \in C^{1,2,2}(\mathcal{O})$, and any fixed $\pi \in \Pi$, the usual infinitesimal generator $\mathscr{A}$ for wealth process (14) is defined as

$$
\begin{aligned}
\mathscr{A}^{\pi} \phi(t, x, m, z)= & \phi_{t}(t, x, m, z)+\left[c-(1-a(t)) c_{1}+a(t) \sigma_{1}\left(\delta_{0}-m\right)+r x+\pi_{1}(t)(\mu-r)+\pi_{2}(t)(1-z) \delta\right] \\
& \cdot \phi_{x}(t, x, m, z)+\frac{1}{2}\left[a^{2}(t) \sigma_{1}^{2}+\pi_{1}^{2}(t) \sigma_{2}^{2}+2 \rho \sigma_{1} \sigma_{2} a(t) \pi_{1}(t)\right] \phi_{x x}(t, x, m, z) \\
& +\left[\frac{a(t) \sigma_{1}}{T_{0}-t}+\frac{\rho \pi_{1}(t) \sigma_{2}}{T_{0}-t}\right] \phi_{x m}(t, x, m, z)+\frac{1}{2\left(T_{0}-t\right)^{2}} \phi_{m m}(t, x, m, z) \\
& +\lambda_{1} E[\phi(t, x-a(t) Y, m, z)-\phi(t, x, m, z)]+\lambda_{2} E\left[\phi\left(t, x+\pi_{1}(t) U, m, z\right)-\phi(t, x, m, z)\right] \\
& +\lambda E\left[\phi\left(t, x-a(t) Y+\pi_{1}(t) U, m, z\right)-\phi(t, x, m, z)\right] \\
& +h^{P}(1-z)\left[\phi\left(t, x-\pi_{2}(t)(1-z) \zeta, m, z+1\right)-\phi(t, x, m, z)\right] .
\end{aligned}
$$

Similar to Theorem 7.1 in the study Bjö rk and Murgoci [7], we present the following verification theorem for the predefault case, i.e., the case $z=0$.
Theorem 2. Verification theorem: if there are two functions $W(t, x, m, 0) \in C^{1,2,2}(\mathcal{O})$ and $g(t, x, m, 0) \in C^{1,2,2}(\mathcal{O})$ satisfying the following extended HJB equations,

$$
\left\{\begin{array}{l}
\sup _{\pi \in \Pi}\left\{\mathscr{A}^{\pi} W(t, x, m, 0)-\mathscr{A}^{\pi} \frac{\gamma}{2} g^{2}(t, x, m, 0)+\gamma g(t, x, m, 0) \mathscr{A}^{\pi} g(t, x, m, 0)\right\}=0, \\
\mathscr{A}^{\pi^{*}} g(t, x, m, 0)=0,0 \leq t \leq T,
\end{array}\right.
$$


with boundary conditions $W(T, x, m, 0)=x$ and $g(T, x$, $m, 0)=x$, where

$$
\pi^{*}=\operatorname{argsup}_{\pi \in \Pi}\left\{\mathscr{A}^{\pi} W(t, x, m, 0)-\mathscr{A}^{\pi} \frac{\gamma}{2} g^{2}(t, x, m, 0)+\gamma g(t, x, m, 0) \mathscr{A}^{\pi} g(t, x, m, 0)\right\} .
$$

Then, $V(t, x, m, 0)=W(t, x, m, 0), E_{t, x, m, 0}\left[X_{T}^{\pi^{*}}\right]=g(t$, $x, m, 0)$, and $\pi^{*}$ is the closed-loop equilibrium strategy.

In the postdefault case, the defaultable bond is not traded and the model framework can be regarded as a special situation of the predefault. Similar to Theorem 2, we present the following verification theorem for the postdefault case.
Theorem 3. Verification theorem: for the postdefault case, i.e., the case $z=1$, the extended HJB equations are defined as follows:

$$
\left\{\begin{array}{l}
\sup _{\pi \in \Pi}\left\{\mathscr{A}^{\pi} W(t, x, m, 1)-\frac{1}{2} \mathscr{A}^{\pi} \gamma g^{2}(t, x, m, 1)+\gamma g(t, x, m, 1) \mathscr{A}^{\pi} \gamma g(t, x, m, 1)\right\}=0, \\
\mathscr{A}^{\pi^{*}} g(t, x, m, 1)=0,0 \leq t \leq T
\end{array}\right.
$$

with boundary conditions $W(T, x, m, 1)=x$ and $g(T, x$, $m, 1)=x$, where

$$
\begin{aligned}
\pi^{*}= & \arg \sup _{\pi \in \Pi}\left\{\mathscr{A}^{\pi} W(t, x, m, 1)-0.5 \mathscr{A}^{\pi} \gamma g^{2}(t, x, m, 1)\right. \\
& \left.+\gamma g(t, x, m, 1) \mathscr{A}^{\pi} \gamma g(t, x, m, 1)\right\} .
\end{aligned}
$$

Then, $V(t, x, m, 1)=W(t, x, m, 1), E_{t, x, m, 1}\left[X_{T}^{\pi^{*}}\right]=g(t$, $x, m, 1)$, and $\pi^{*}$ is the closed-loop equilibrium strategy.

\section{The Main Result}

This section is devoted to obtain the closed-loop equilibrium reinsurance-investment strategy and the corresponding value function for problem (16) in the postdefault case $(z=$ $1)$ and the predefault case $(z=0)$, respectively.

4.1. Postdefault Case: $z=1$. In this section, we derive the closed-loop equilibrium reinsurance-investment strategy in the postdefault case. After default, we have $P\left(t, T_{1}\right)=0$ for $\tau \leq t \leq T$ and then $\pi_{2}(t)=0$ for $\tau \leq t \leq T$. Assume that there exist two functions $W(t, x, m, 1)$ and $g(t, x, m, 1)$ satisfy Theorem 3. Then, the first equation of equation (25) can be rewritten as

$$
\begin{aligned}
& \sup _{\pi \in \Pi}\left\{W_{t}(t, x, m, 1)+\left[r x+c-c_{1}+a(t)\left(c_{1}+\sigma_{1}\left(\delta_{0}-m\right)\right)+\pi_{1}(t)(\mu-r)\right] W_{x}(t, x, m, 1)\right. \\
& +\frac{1}{2}\left[a^{2}(t) \sigma_{1}^{2}+\pi_{1}^{2}(t) \sigma_{2}^{2}+2 \rho \sigma_{1} \sigma_{2} a(t) \pi_{1}(t)\right]\left[W_{x x}(t, x, m, 1)-\gamma g_{x}^{2}(t, x, m, 1)\right] \\
& +\left[\frac{a(t) \sigma_{1}}{T_{0}-t}+\frac{\rho \pi_{1}(t) \sigma_{2}}{T_{0}-t}\right]\left[W_{x m}(t, x, m, 1)-\gamma g_{x}(t, x, m, 1) g_{m}(t, x, m, 1)\right] \\
& +\frac{1}{2\left(T_{0}-t\right)^{2}}\left[W_{m m}(t, x, m, 1)-\gamma g_{m}^{2}(t, x, m, 1)\right]+\lambda_{1} E[W(t, x-a(t) Y, m, 1)-W(t, x, m, 1)] \\
& \quad-0.5 \lambda_{1} \gamma E\left\{[g(t, x-a(t) Y, m, 1)-g(t, x, m, 1)]^{2}\right\}+\lambda_{2} E\left[W\left(t, x+\pi_{1}(t) U, m, 1\right)-W(t, x, m, 1)\right] \\
& -0.5 \lambda_{2} \gamma E\left\{\left[g\left(t, x-\pi_{1}(t) U, m, 1\right)-g(t, x, m, 1)\right]^{2}\right\}+\lambda E\left[W\left(t, x-a(t) Y+\pi_{1}(t) U, m, 1\right)-W(t, x, m, 1)\right] \\
& \left.-0.5 \lambda \gamma E\left\{\left[g\left(t, x-a(t) Y+\pi_{1}(t), m, 1\right)-g(t, x, m, 1)\right]^{2}\right\}\right\}=0 .
\end{aligned}
$$


According to the the second equation of equations (25) and (27), and the boundary conditions $W(T, x, m, 1)=x$ and $g(T, x, m, 1)=x$, we conjecture that the solutions to the second equation of (25) and (27) have the following forms:

$$
\left\{\begin{array}{l}
g(t, x, m, 1)=\bar{A}_{1}(t) x+\bar{B}_{1}(t) m^{2}+\bar{L}_{1}(t) m+\bar{N}_{1}(t), \\
W(t, x, m, 1)=A_{1}(t) x+B_{1}(t) m^{2}+L_{1}(t) m+N_{1}(t),
\end{array}\right.
$$

with boundary conditions

$$
\left\{\begin{array}{l}
\bar{A}_{1}(T)=1, \bar{B}_{1}(T)=\bar{L}_{1}(T)=\bar{N}_{1}(T)=0, \\
A_{1}(T)=1, B_{1}(T)=L_{1}(T)=N_{1}(T)=0 .
\end{array}\right.
$$

The corresponding partial derivatives are given by

$$
\left\{\begin{array}{l}
W_{t}(t, x, m, 1)=A_{1}^{\prime}(t) x+B_{1}^{\prime}(t) m^{2}+L_{1}^{\prime}(t) m+N_{1}^{\prime}(t), \\
W_{x}(t, x, m, 1)=A_{1}(t), \\
W_{x x}(t, x, m, 1)=0, \\
W_{m}(t, x, m, 1)=2 B_{1}(t) m+L_{1}(t), \\
W_{m m}(t, x, m, 1)=2 B_{1}(t), \\
W_{x m}(t, x, m, 1)=0, \\
g_{t}(t, x, m, 1)=\bar{A}_{1}^{\prime}(t) x+\bar{B}_{1}^{\prime}(t) m^{2}+\bar{L}_{1}^{\prime}(t) m+\bar{N}_{1}^{\prime}(t), \\
g_{x}(t, x, m, 1)=\bar{A}_{1}(t), \\
g_{x x}(t, x, m, 1)=0 \\
g_{m}(t, x, m, 1)=2 \bar{B}_{1}(t) m+\bar{L}_{1}(t), \\
g_{m m}(t, x, m, 1)=2 \bar{B}_{1}(t), \\
g_{x m}(t, x, m, 1)=0 .
\end{array}\right.
$$

Plugging (28) and the above partial derivatives into (27), we obtain after simplification

$$
\begin{aligned}
& {\left[A_{1}^{\prime}(t)+r A_{1}(t)\right] x+B_{1}^{\prime}(t) m^{2}+L_{1}^{\prime}(t) m} \\
& \quad+N_{1}^{\prime}(t)+\left(c-c_{1}\right) A_{1}(t) \\
& \quad+\frac{1}{2\left(T_{0}-t\right)^{2}}\left[2 B_{1}(t)-\gamma\left(2 \bar{B}_{1}(t) m+\bar{L}_{1}(t)\right)^{2}\right] \\
& \quad+\sup _{\pi \in \Pi} \mathscr{B}\left(a(t), \pi_{1}(t)\right)=0,
\end{aligned}
$$

where

$$
\begin{aligned}
\mathscr{B}\left(a(t), \pi_{1}(t)\right)= & -\frac{\gamma}{2}\left[\sigma_{1}^{2}+\left(\lambda_{1}+\lambda\right) \mu_{12}\right] \bar{A}_{1}^{2}(t) a^{2}(t) \\
& +\left\{\left[c_{1}+\sigma_{1}\left(\delta_{0}-m\right)-\left(\lambda_{1}+\lambda\right) \mu_{11}\right] A_{1}(t)\right. \\
& \left.-\frac{a(t) \sigma_{1}}{T_{0}-t}\left[2 \bar{B}_{1}(t) m+\bar{L}_{1}(t)\right] \gamma \bar{A}_{1}(t)\right\} a(t) \\
& -\frac{\gamma}{2}\left[\sigma_{2}^{2}+\left(\lambda_{2}+\lambda\right) \mu_{22}\right] \bar{A}_{1}^{2}(t) \pi_{1}^{2}(t) \\
& +\left\{\left[\mu-r+\left(\lambda_{2}+\lambda\right) \mu_{21}\right] A_{1}(t)-\frac{\rho \sigma_{2}}{T_{0}-t}\right. \\
& \left.\cdot\left[2 \bar{B}_{1}(t) m+\bar{L}_{1}(t)\right] \gamma \bar{A}_{1}(t)\right\} \pi_{1}(t) \\
& +\left(\lambda \mu_{11} \mu_{21}-\rho \sigma_{1} \sigma_{2}\right) \gamma a(t) \pi_{1}(t) \bar{A}_{1}^{2}(t) .
\end{aligned}
$$

Setting $\left(\partial \mathscr{B}\left(a(t), \pi_{1}(t)\right) / \partial a(t)\right)=0$ and $\left(\partial \mathscr{B}\left(a(t), \pi_{1}\right.\right.$ $\left.(t)) / \partial \pi_{1}(t)\right)=0$, we obtain

$$
\left\{\begin{array}{l}
{\left[\sigma_{1}^{2}+\left(\lambda_{1}+\lambda\right) \mu_{12}\right] \gamma \bar{A}_{1}^{2}(t) a(t)-\left(\lambda \mu_{11} \mu_{21}-\rho \sigma_{1} \sigma_{2}\right) \gamma \bar{A}_{1}^{2}(t) \pi_{1}(t)} \\
=\left[c_{1}+\sigma_{1}\left(\delta_{0}-m\right)-\left(\lambda_{1}+\lambda\right) \mu_{11}\right] A_{1}(t)-\frac{\sigma_{1}}{T_{0}-t}\left[2 \bar{B}_{1}(t) m+\bar{L}_{1}(t)\right] \gamma \bar{A}_{1}(t), \\
-\left(\lambda \mu_{11} \mu_{21}-\rho \sigma_{1} \sigma_{2}\right) \gamma \bar{A}_{1}^{2}(t) a(t)+\left[\sigma_{2}^{2}+\left(\lambda_{2}+\lambda\right) \mu_{22}\right] \gamma \bar{A}_{1}^{2}(t) \pi_{1}(t) \\
=\left[\mu-r+\left(\lambda_{2}+\lambda\right) \mu_{21}\right] A_{1}(t)-\frac{\rho \sigma_{2}}{T_{0}-t}\left[2 \bar{B}_{1}(t) m+\bar{L}_{1}(t)\right] \gamma \bar{A}_{1}(t) .
\end{array}\right.
$$


Equation (33) is a system of linear equations with respect to $a(t)$ and $\pi_{1}(t)$, and the determinant of its coefficient matrix is

$$
\begin{aligned}
& \left|\begin{array}{l}
{\left[\sigma_{1}^{2}+\left(\lambda_{1}+\lambda\right) \mu_{12}\right] \gamma \bar{A}_{1}^{2}(t)-\left(\lambda \mu_{11} \mu_{21}-\rho \sigma_{1} \sigma_{2}\right) \gamma \bar{A}_{1}^{2}(t)} \\
-\left(\lambda \mu_{11} \mu_{21}-\rho \sigma_{1} \sigma_{2}\right) \gamma \bar{A}_{1}^{2}(t)\left[\sigma_{2}^{2}+\left(\lambda_{2}+\lambda\right) \mu_{22}\right] \gamma \bar{A}_{1}^{2}(t)
\end{array}\right| \\
& \quad=\left\{\left[\sigma_{1}^{2}+\left(\lambda_{1}+\lambda\right) \mu_{12}\right]\left[\sigma_{2}^{2}+\left(\lambda_{2}+\lambda\right) \mu_{22}\right]\right. \\
& \left.\quad-\left(\lambda \mu_{11} \mu_{21}-\rho \sigma_{1} \sigma_{2}\right)^{2}\right\} \gamma^{2} \bar{A}_{1}^{4}(t) \\
& \quad>\left(\mu_{12} \mu_{22}-\mu_{11} \mu_{21}\right) \gamma^{2} \bar{A}_{1}^{4}(t) .
\end{aligned}
$$

By the Cauchy-Schwarz inequality, it is clear that $\mu_{12} \mu_{22}-\mu_{11} \mu_{21}>0$. Therefore, equation (33) has a unique root $\left(\widehat{a}(t) t, n \widehat{\pi}_{1} q(t)\right)$ is given by

$$
\begin{aligned}
\{\hat{a}(t) & =\frac{q_{6} A_{1}(t)-q_{7}(t) \bar{A}_{1}(t) \bar{L}_{1}(t)-\left[\sigma_{1} q_{2} A_{1}(t)+2 q_{7}(t) \bar{A}_{1}(t) \bar{B}_{1}(t)\right] m}{\left(q_{1} q_{2}-q_{3}^{2}\right) \gamma \bar{A}_{1}^{2}(t)}, \\
\hat{\pi}_{1}(t) & =\frac{q_{8} A_{1}(t)-q_{9}(t) \bar{A}_{1}(t) \bar{L}_{1}(t)-\left[\sigma_{1} q_{3} A_{1}(t)+2 q_{9}(t) \bar{A}_{1}(t) \bar{B}_{1}(t)\right] m}{\left(q_{1} q_{2}-q_{3}^{2}\right) \gamma \bar{A}_{1}^{2}(t)},
\end{aligned}
$$

where

$$
\left\{\begin{array}{l}
q_{1}=\sigma_{1}^{2}+\left(\lambda_{1}+\lambda\right) \mu_{12}, \\
q_{2}=\sigma_{2}^{2}+\left(\lambda_{2}+\lambda\right) \mu_{22}, \\
q_{3}=\lambda \mu_{11} \mu_{21}-\rho \sigma_{1} \sigma_{2}, \\
q_{4}=\mu-r+\left(\lambda_{2}+\lambda\right) \mu_{21}, \\
q_{5}=c_{1}+\sigma_{1} \delta_{0}-\left(\lambda_{1}+\lambda\right) \mu_{11}, \\
q_{6}=q_{2} q_{5}+q_{3} q_{4}, \\
q_{7}(t)=\frac{\sigma_{1} \gamma q_{2}+\rho \sigma_{2} \gamma q_{3}}{T_{0}-t}, \\
q_{8}=q_{1} q_{4}+q_{3} q_{5}, \\
q_{9}(t)=\frac{\rho \sigma_{2} \gamma q_{1}+\sigma_{1} \gamma q_{3}}{T_{0}-t} .
\end{array}\right.
$$

We now study the closed-loop equilibrium reinsurance strategy for the following two cases.

4.1.1. $\hat{a}(t)>0$. In this section, we assume that $A_{1}(t)=A_{11}(t), \quad B_{1}(t)=B_{11}(t), \quad L_{1}(t)=L_{11}(t), \quad N_{1}(t)=$ $N_{11}(t), \bar{A}_{1}(t)=\bar{A}_{11}(t), \bar{B}_{1}(t)=\bar{B}_{11}(t), \bar{L}_{1}(t)=\bar{L}_{11}(t)$, and $\bar{N}_{1}(t)=\bar{N}_{11}(t)$. Furthermore, from (35), we can obtain that the closed-loop equilibrium reinsurance and investment strategies are, respectively, given by

$$
\begin{aligned}
& \left\{\frac{a^{*}(t)=q_{6} A_{11}(t)-q_{7}(t) \bar{A}_{11}(t) \bar{L}_{11}(t)-\left[\sigma_{1} q_{2} A_{11}(t)+2 q_{7}(t) \bar{A}_{11}(t) \bar{B}_{11}(t)\right] m}{\left(q_{1} q_{2}-q_{3}^{2}\right) \gamma \bar{A}_{11}^{2}(t)},\right. \\
& \pi_{1}^{*}(t)=\frac{q_{8} A_{11}(t)-q_{9}(t) \bar{A}_{11}(t) \bar{L}_{11}(t)-\left[\sigma_{1} q_{3} A_{11}(t)+2 q_{9}(t) \bar{A}_{11}(t) \bar{B}_{11}(t)\right] m}{\left(q_{1} q_{2}-q_{3}^{2}\right) \gamma \bar{A}_{11}^{2}(t)} .
\end{aligned}
$$


For presentation convenience, we introduce the following notations

$$
\begin{aligned}
& \left\{\begin{array}{l}
\mathcal{O}_{1}=\left\{t \mid q_{6}-q_{7}(t) \bar{L}_{11}(t)-\left[\sigma_{1} q_{2}+2 q_{7}(t) \bar{B}_{11}(t)\right] m>0\right\} \\
\mathcal{O}_{2}=\left\{t \mid q_{6}-q_{7}(t) \bar{L}_{11}(t)-\left[\sigma_{1} q_{2}+2 q_{7}(t) \bar{B}_{11}(t)\right] m \leq 0\right\} \\
\Gamma_{11}(t)=\frac{q_{6} A_{11}(t)-q_{7}(t) \bar{A}_{11}(t) \bar{L}_{11}(t)}{\left(q_{1} q_{2}-q_{3}^{2}\right) \gamma \bar{A}_{11}^{2}(t)} \\
\Gamma_{21}(t)=\frac{\sigma_{1} q_{2} A_{11}(t)+2 q_{7}(t) \bar{A}_{11}(t) \bar{B}_{11}(t)}{\left(q_{1} q_{2}-q_{3}^{2}\right) \gamma \bar{A}_{11}^{2}(t)} \\
\Gamma_{31}(t)=\frac{q_{8} A_{11}(t)-q_{9}(t) \bar{A}_{11}(t) \bar{L}_{11}(t)}{\left(q_{1} q_{2}-q_{3}^{2}\right) \gamma \bar{A}_{11}^{2}(t)} \\
\Gamma_{41}(t)=\frac{\sigma_{1} q_{3} A_{11}(t)+2 q_{9}(t) \bar{A}_{11}(t) \bar{B}_{11}(t)}{\left(q_{1} q_{2}-q_{3}^{2}\right) \gamma \bar{A}_{11}^{2}(t)} \\
\Gamma_{81}(t)=\sigma_{1} A_{11}(t)+\frac{2 \sigma_{1} \gamma}{T_{0}-t} \bar{A}_{11}(t) \bar{B}_{11}(t) \\
\Gamma_{51}(t)=q_{4} A_{11}(t)-\frac{\rho \sigma_{2} \gamma}{T_{0}-t} \bar{A}_{11}(t) \bar{L}_{11}(t) \\
\Gamma_{71}(t)=q_{5} A_{11}(t), \\
\Gamma_{0}-t \\
\Gamma_{11}(t) \bar{B}_{11}(t)
\end{array}\right.
\end{aligned}
$$

Plugging (37) into (31), we obtain after simplification

$$
\begin{aligned}
& {\left[A_{11}^{\prime}(t)+r A_{11}(t)\right] x} \\
& \quad+\left\{B_{11}^{\prime}(t)-\frac{2 \gamma \bar{B}_{11}^{2}(t)}{\left(T_{0}-t\right)^{2}}-\frac{\gamma q_{2}}{2} \bar{A}_{11}^{2}(t) \Gamma_{41}^{2}(t)-\frac{\gamma q_{1}}{2} \bar{A}_{11}^{2}(t) \Gamma_{21}^{2}(t)+\gamma q_{5} \bar{A}_{11}^{2}(t) \Gamma_{21}(t) \Gamma_{41}(t)+\Gamma_{21}(t) \Gamma_{81}(t)+\Gamma_{41}(t) \Gamma_{61}(t)\right\} m^{2} \\
& \quad+\left\{\frac{L_{11}^{\prime}(t)-2 \gamma \bar{B}_{11}(t) \bar{L}_{11}(t)}{\left(T_{0}-t\right)^{2}-\frac{\gamma q_{2}}{2} \bar{A}_{11}^{2}(t) \Gamma_{31}(t) \Gamma_{41}(t)-\frac{\gamma q_{1}}{2} \bar{A}_{11}^{2}(t) \Gamma_{11}(t) \Gamma_{21}(t)}\right. \\
& \quad-\gamma q_{5} \bar{A}_{11}^{2}(t)\left[\Gamma_{21}(t) \Gamma_{31}(t)+\Gamma_{11}(t) \Gamma_{41}(t)\right]-\Gamma_{11}(t) \Gamma_{81}(t)-\Gamma_{31}(t) \Gamma_{61}(t)-\Gamma_{41}(t) \Gamma_{51}(t) \\
& \left.\quad-\Gamma_{21}(t) \Gamma_{71}(t)\right\} m+\left\{N_{11}^{\prime}(t)+\left(c-c_{1}\right) A_{11}(t)+\frac{B_{11}(t)}{\left(T_{0}-t\right)^{2}}-\frac{\gamma \bar{L}_{11}^{2}(t)}{2\left(T_{0}-t\right)^{2}}-\frac{\gamma q_{2}}{2} \bar{A}_{11}^{2}(t) \Gamma_{31}^{2}(t)\right. \\
& \left.\quad-\frac{\gamma q_{1}}{2} \bar{A}_{11}^{2}(t) \Gamma_{11}^{2}(t)+\gamma q_{5} \bar{A}_{11}^{2}(t) \Gamma_{11}(t) \Gamma_{31}(t)+\Gamma_{11}(t) \Gamma_{71}(t)+\Gamma_{31}(t) \Gamma_{51}(t)\right\}=0 .
\end{aligned}
$$


By separating the variables $x, m$, and $m^{2}$, we derive the following ordinary differential equations with associated boundary conditions

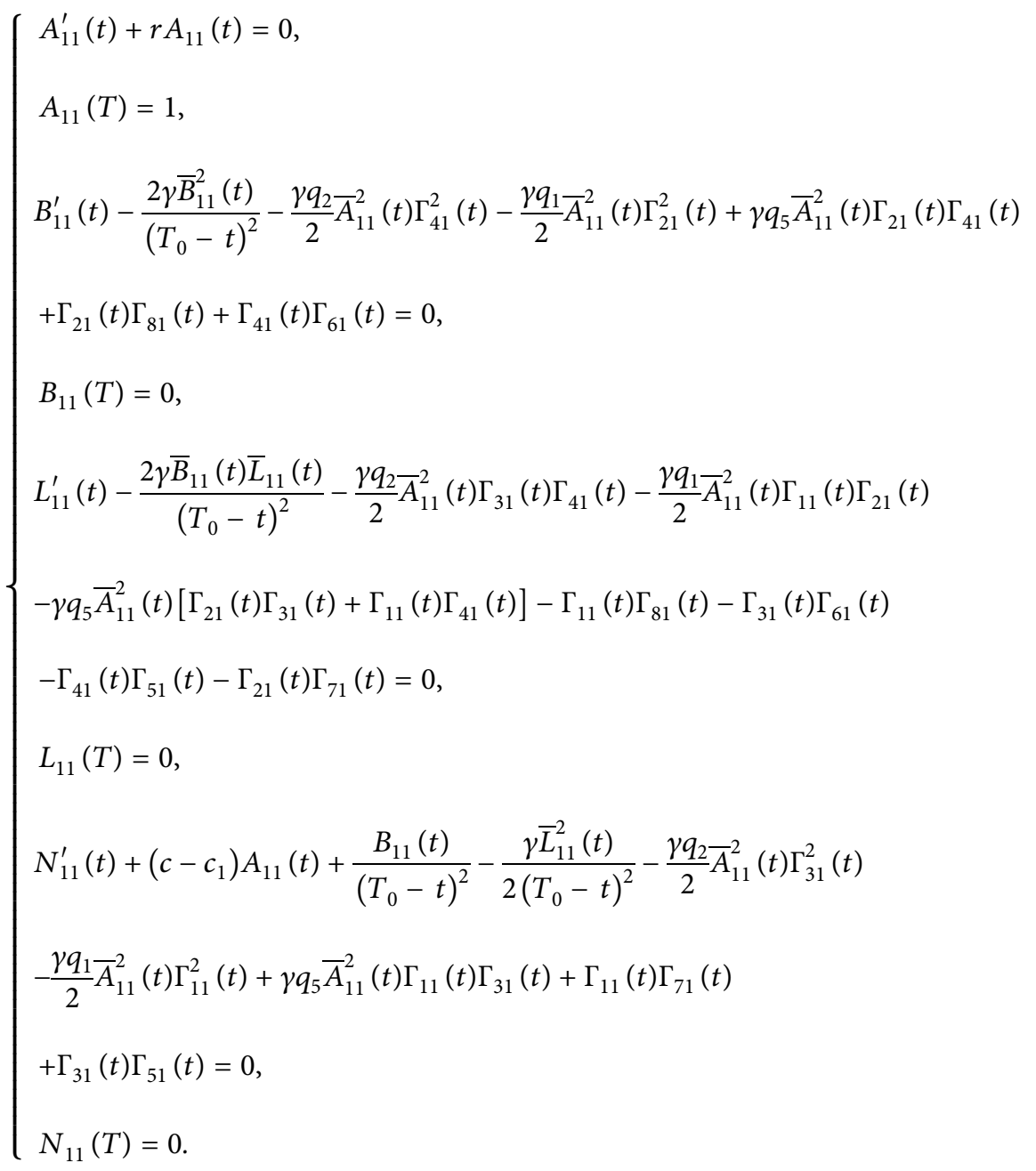

By substituting (37) into the second equation of equation (25), we obtain

$$
\begin{aligned}
& {\left[\bar{A}_{11}^{\prime}(t)+r \bar{A}_{11}(t)\right] x} \\
& \quad+\left\{\bar{B}_{11}^{\prime}(t)+\frac{2 \sigma_{1} q_{7}(t) \bar{B}_{11}(t)}{\left(q_{1} q_{2}-q_{3}^{2}\right) \gamma}+\frac{\sigma_{1}^{2} q_{2} A_{11}(t)}{\left(q_{1} q_{2}-q_{3}^{2}\right) \gamma \bar{A}_{11}(t)}\right\} m^{2} \\
& \quad+\left\{\bar{L}_{11}^{\prime}(t)+\frac{\sigma_{1} q_{7}(t) \bar{L}_{11}(t)}{\left(q_{1} q_{2}-q_{3}^{2}\right) \gamma}-\frac{\left(\sigma_{1} q_{6}+q_{4} \sigma_{1} q_{3}+q_{5} \sigma_{1} q_{2}\right) A_{11}(t)}{\left(q_{1} q_{2}-q_{3}^{2}\right) \gamma \bar{A}_{11}(t)}-\frac{\left[2 q_{4} q_{9}(t)+q_{5} q_{8}\right] \bar{B}_{11}(t)}{\left(q_{1} q_{2}-q_{3}^{2}\right) \gamma}\right\} m \\
& \quad+\left\{\bar{N}_{11}^{\prime}(t)+\left(c-c_{1}\right) \bar{A}_{11}(t)+\frac{\bar{B}_{11}(t)}{\left(T_{0}-t\right)^{2}}-\frac{\left[q_{4} q_{9}(t)+q_{5} q_{7}(t)\right] \bar{L}_{11}(t)}{\left(q_{1} q_{2}-q_{3}^{2}\right) \gamma}+\frac{\left(q_{4} q_{9}+q_{5} q_{6}\right) A_{11}(t)}{\left(q_{1} q_{2}-q_{3}^{2}\right) \gamma \bar{A}_{11}(t)}\right\}=0
\end{aligned}
$$


By separating the variables $x, m$, and $m^{2}$, we derive the following ordinary differential equations with associated boundary conditions

$$
\begin{aligned}
& \int \bar{A}_{11}^{\prime}(t)+r \bar{A}_{11}(t)=0, \bar{A}_{11}(T)=1, \\
& \bar{B}_{11}^{\prime}(t)+\frac{2 \sigma_{1} q_{7}(t) \bar{B}_{11}(t)}{\left(q_{1} q_{2}-q_{3}^{2}\right) \gamma}+\frac{\sigma_{1}^{2} q_{2} A_{11}(t)}{\left(q_{1} q_{2}-q_{3}^{2}\right) \gamma \bar{A}_{11}(t)}=0 \\
& \bar{B}_{11}(T)=0 \text {, } \\
& \bar{L}_{11}^{\prime}(t)+\frac{\sigma_{1} q_{7}(t) \bar{L}_{11}(t)}{\left(q_{1} q_{2}-q_{3}^{2}\right) \gamma}-\frac{\left(\sigma_{1} q_{6}+q_{4} \sigma_{1} q_{3}+q_{5} \sigma_{1} q_{2}\right) A_{11}(t)}{\left(q_{1} q_{2}-q_{3}^{2}\right) \gamma \bar{A}_{11}(t)} \\
& -\frac{\left[2 q_{4} q_{9}(t)+q_{5} q_{8}\right] \bar{B}_{11}(t)}{\left(q_{1} q_{2}-q_{3}^{2}\right) \gamma}=0, \\
& \bar{L}_{11}(T)=0, \\
& \bar{N}_{11}^{\prime}(t)+\left(c-c_{1}\right) \bar{A}_{11}(t)+\frac{\bar{B}_{11}(t)}{\left(T_{0}-t\right)^{2}}-\frac{\left.\left[q_{4} q_{9}(t)+q_{5} q_{7}(t)\right] \bar{L}_{11}(t)\right)}{\left(q_{1} q_{2}-q_{3}^{2}\right) \gamma} \\
& +\frac{\left(q_{4} q_{9}(t)+q_{5} q_{6}\right) A_{11}(t)}{\left(q_{1} q_{2}-q_{3}^{2}\right) \gamma \bar{A}_{11}(t)}=0 \\
& \bar{N}_{11}(T)=0 .
\end{aligned}
$$


By solving the above equations ((40) and (42)), we have

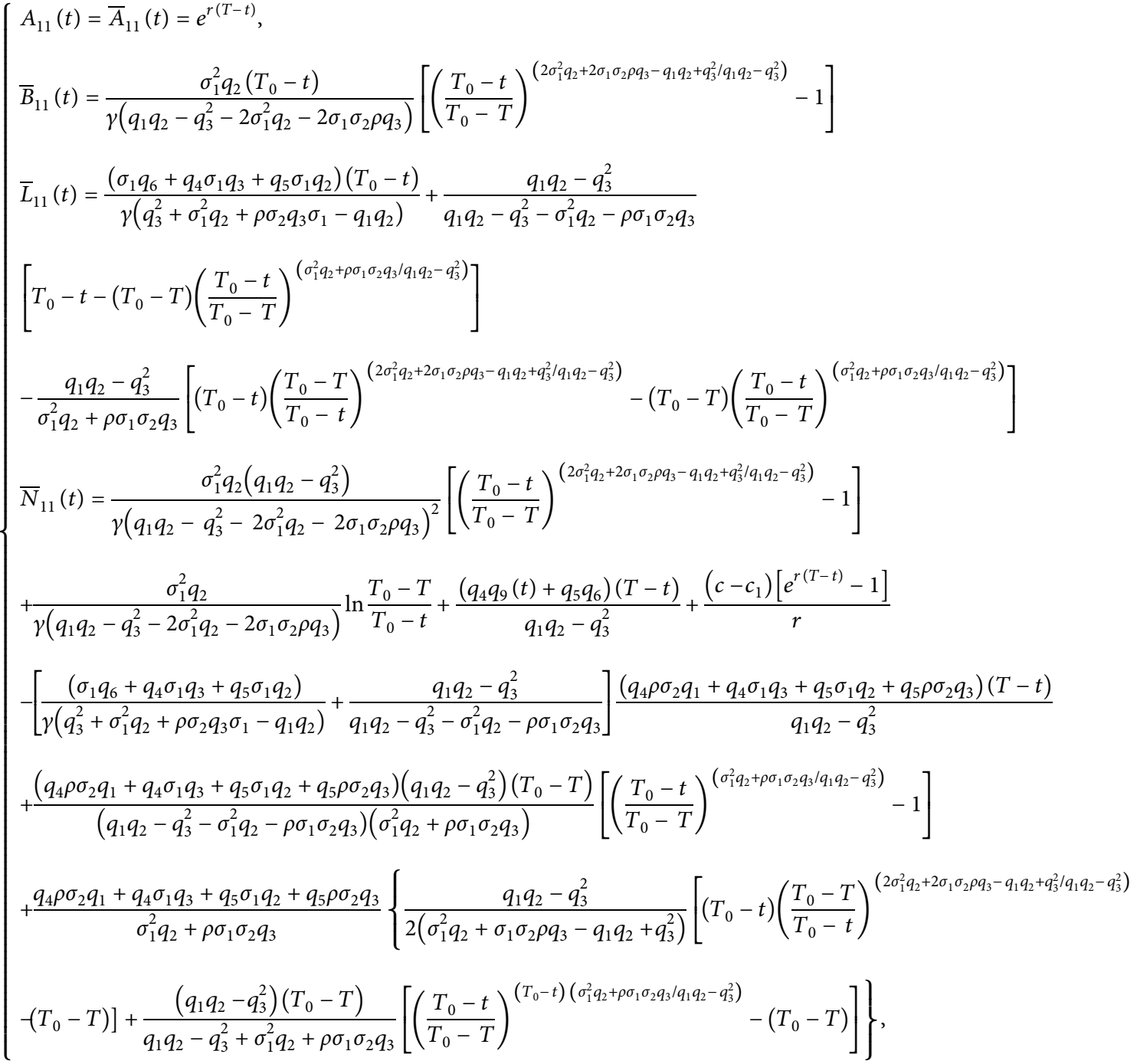

$$
\begin{aligned}
& B_{11}(t)=\int_{t}^{T}\left\{\gamma q_{5} \bar{A}_{11}^{2}(s) \Gamma_{21}(s) \Gamma_{41}(s)+\Gamma_{21}(s) \Gamma_{81}(s)+\Gamma_{41}(s) \Gamma_{61}(s)-\frac{2 \gamma \bar{B}_{11}^{2}(s)}{\left(T_{0}-s\right)^{2}}\right. \\
& \left.-\frac{\gamma q_{2}}{2} \bar{A}_{11}^{2}(s) \Gamma_{41}^{2}(s)-\frac{\gamma q_{1}}{2} \bar{A}_{11}^{2}(s) \Gamma_{21}^{2}(s)\right\} \mathrm{d} s, \\
& L_{11}(t)=-\int_{t}^{T}\left\{\frac{2 \gamma \bar{B}_{1}(s) \bar{L}_{11}(s)}{\left(T_{0}-s\right)^{2}-\frac{\gamma q_{2}}{2} \bar{A}_{11}^{2}(s) \Gamma_{31}(s) \Gamma_{41}(s)-\frac{\gamma q_{1}}{2} \bar{A}_{11}^{2}(s) \Gamma_{11}(s) \Gamma_{21}(s)}\right. \\
& -\gamma q_{5} \bar{A}_{11}^{2}(s)\left[\Gamma_{21}(t) \Gamma_{31}(s)+\Gamma_{11}(s) \Gamma_{41}(s)\right]-\Gamma_{11}(s) \Gamma_{81}(s)-\Gamma_{31}(s) \Gamma_{61}(s) \\
& \left.-\Gamma_{41}(s) \Gamma_{51}(s)-\Gamma_{21}(s) \Gamma_{71}(s)\right\} \mathrm{d} s, \\
& N_{11}(t)=\int_{t}^{T}\left\{\left(c-c_{1}\right) A_{11}(t)+\frac{B_{11}(t)}{\left(T_{0}-t\right)^{2}}-\frac{\gamma \bar{L}_{11}^{2}(t)}{2\left(T_{0}-t\right)^{2}}-\frac{\gamma q_{2}}{2} \bar{A}_{11}^{2}(t) \Gamma_{31}^{2}(t)\right. \\
& -\frac{\gamma q_{1}}{2} \bar{A}_{11}^{2}(t) \Gamma_{11}^{2}(t)+\gamma q_{5} \bar{A}_{11}^{2}(t) \Gamma_{11}(t) \Gamma_{31}(t)+\Gamma_{11}(t) \Gamma_{71}(t) \\
& \left.+\Gamma_{31}(t) \Gamma_{51}(t)\right\} \mathrm{d} s .
\end{aligned}
$$


4.1.2. $\hat{a}(t) \leq 0$. In this section, we assume that $A_{1}(t)=A_{12}(t), \quad B_{1}(t)=B_{12}(t), \quad L_{1}(t)=L_{12}(t), \quad N_{1}(t)=$ $N_{12}(t), \bar{A}_{1}(t)=\bar{A}_{12}(t), \bar{B}_{1}(t)=\bar{B}_{12}(t), \bar{L}_{1}(t)=\bar{L}_{12}(t)$, and
$\bar{N}_{1}(t)=\bar{N}_{12}(t)$. For presentation convenience, we introduce the following notations:

$$
\left\{\begin{array}{l}
\Gamma_{12}(t)=\frac{q_{6} A_{12}(t)-q_{7}(t) \bar{A}_{12}(t) \bar{L}_{12}(t)}{\left(q_{1} q_{2}-q_{3}^{2}\right) \gamma \bar{A}_{12}^{2}(t)}, \\
\Gamma_{22}(t)=\frac{\sigma_{1} q_{2} A_{12}(t)+2 q_{7}(t) \bar{A}_{12}(t) \bar{B}_{11}(t)}{\left(q_{1} q_{2}-q_{3}^{2}\right) \gamma \bar{A}_{12}^{2}(t)}, \\
\Gamma_{32}(t)=\frac{q_{8} A_{12}(t)-q_{9}(t) \bar{A}_{12}(t) \bar{L}_{12}(t)}{\left(q_{1} q_{2}-q_{3}^{2}\right) \gamma \bar{A}_{12}^{2}(t)} \\
\Gamma_{42}(t)=\frac{\sigma_{1} q_{3} A_{12}(t)+2 q_{9}(t) \bar{A}_{12}(t) \bar{B}_{11}(t)}{\left(q_{1} q_{2}-q_{3}^{2}\right) \gamma \bar{A}_{12}^{2}(t)} \\
\Gamma_{52}(t)=q_{4} A_{12}(t)-\frac{\rho \sigma_{2} \gamma}{T_{0}-t} \bar{A}_{12}(t) \bar{L}_{12}(t), \\
\Gamma_{62}(t)=\frac{2 \rho \sigma_{2} \gamma}{T_{0}-t} \bar{A}_{12}(t) \bar{B}_{12}(t), \\
\Gamma_{72}(t)=q_{5} A_{12}(t), \\
\Gamma_{82}(t)=\sigma_{1} A_{12}(t)+\frac{2 \sigma_{1} \gamma}{T_{0}-t} \bar{A}_{12}(t) \bar{B}_{12}(t) .
\end{array}\right.
$$

Since $\hat{a} \leq 0$, we obtain the closed-loop equilibrium reinsurance strategy is $a^{*}(t)=0$.From (35), the closed-loop equilibrium investment strategy $\pi_{1}^{*}(t)$ is given by

$$
\pi_{1}^{*}(t)=\frac{q_{8} A_{12}(t)-q_{9}(t) \bar{A}_{12}(t) \bar{L}_{12}(t)-\left[\sigma_{1} q_{3} A_{12}(t)+2 q_{9}(t) \bar{A}_{12}(t) \bar{B}_{12}(t)\right] m}{\left(q_{1} q_{2}-q_{3}^{2}\right) \gamma \bar{A}_{12}^{2}(t)} .
$$

Plugging $a^{*}(t)=0$ and (45) into (31), we obtain after simplification

$$
\begin{aligned}
& {\left[A_{12}^{\prime}(t)+r A_{12}(t)\right] x} \\
& \quad+\left\{B_{12}^{\prime}(t)-\frac{2 \gamma \bar{B}_{12}^{2}(t)}{\left(T_{0}-t\right)^{2}}-\frac{\gamma q_{2}}{2} \bar{A}_{12}^{2}(t) \Gamma_{42}^{2}(t)+\Gamma_{42}(t) \Gamma_{62}(t)\right\} m^{2} \\
& \quad+\left\{L_{12}^{\prime}(t)-\frac{2 \gamma \bar{B}_{12}(t) \bar{L}_{12}(t)}{\left(T_{0}-t\right)^{2}}-\gamma q_{2} \bar{A}_{12}^{2}(t) \Gamma_{32}(t) \Gamma_{42}(t)-\Gamma_{32}(t) \Gamma_{62}(t)-\Gamma_{42}(t) \Gamma_{52}(t)\right\} m \\
& \quad+\left\{N_{12}^{\prime}(t)+\left(c-c_{1}\right) A_{12}(t)+\frac{B_{12}(t)}{\left(T_{0}-t\right)^{2}}-\frac{\gamma \bar{L}_{12}^{2}}{2\left(T_{0}-t\right)^{2}}-\frac{\gamma q_{2}}{2} \bar{A}_{12}^{2}(t) \Gamma_{32}^{2}(t)+\Gamma_{32}(t) \Gamma_{52}(t)\right\}=0
\end{aligned}
$$


By separating the variables $x, m$, and $m^{2}$, we derive the following ordinary differential equations with associated boundary conditions

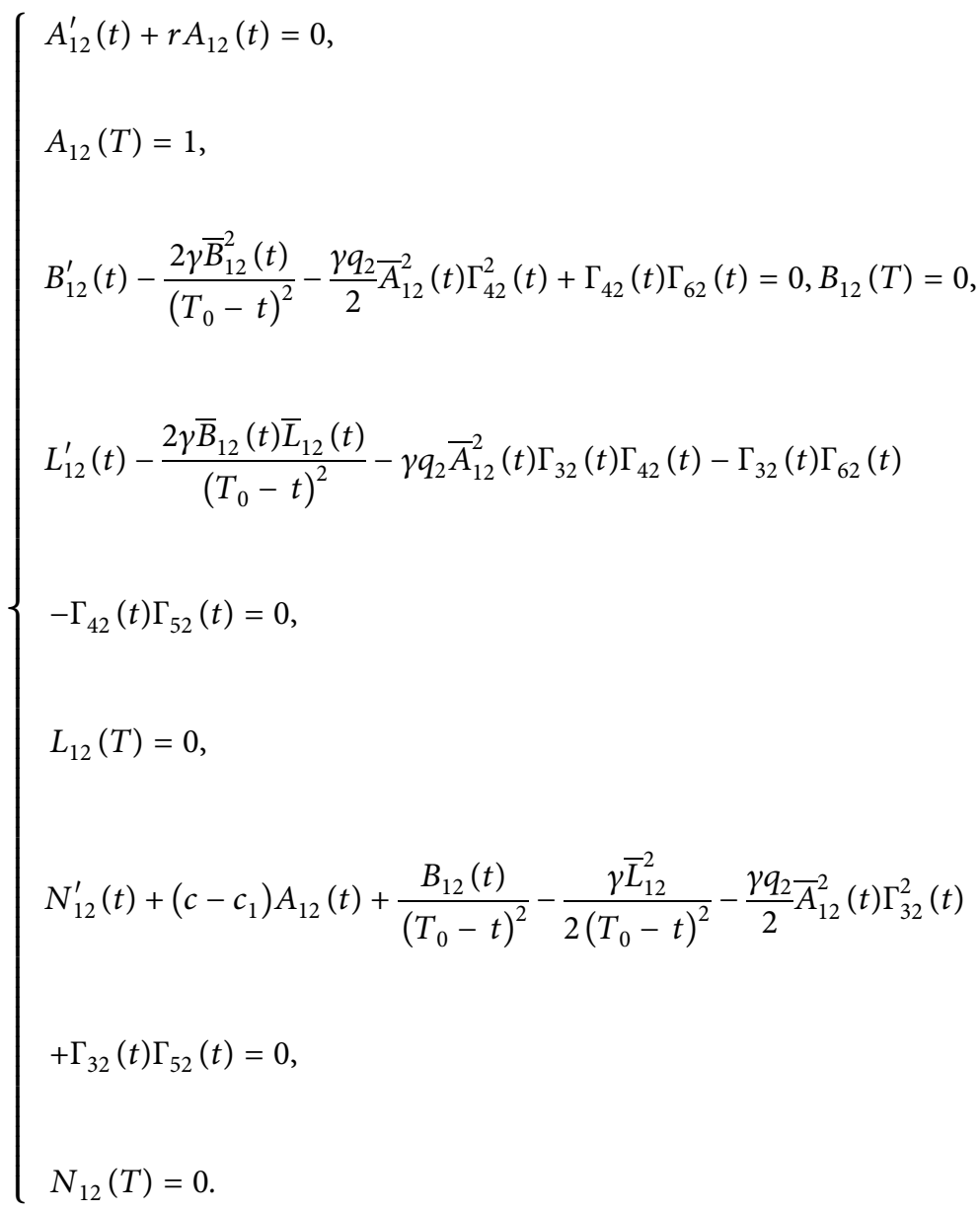

By substituting $a^{*}(t)=0$ and (45) into the second equation of equation (25), we obtain

$$
\begin{aligned}
& {\left[\bar{A}_{12}^{\prime}(t)+r \bar{A}_{12}(t)\right] x+\bar{B}_{12}^{\prime}(t) m^{2}} \\
& \quad+\left\{\bar{L}_{12}^{\prime}(t)-\frac{q_{4} \sigma_{1} q_{3} A_{12}(t)}{\left(q_{1} q_{2}-q_{3}^{2}\right) \gamma \bar{A}_{12}(t)}-\frac{2 q_{4} q_{9}(t) \bar{B}_{12}(t)}{\left(q_{1} q_{2}-q_{3}^{2}\right) \gamma}\right\} m \\
& \quad+\left\{\bar{N}_{12}^{\prime}(t)+\left(c-c_{1}\right) \bar{A}_{12}(t)+\frac{\bar{B}_{12}(t)}{\left(T_{0}-t\right)^{2}}-\frac{q_{4} q_{9}(t) \bar{L}_{12}(t)}{\left(q_{1} q_{2}-q_{3}^{2}\right) \gamma}+\frac{q_{4} q_{8} A_{12}(t)}{\left(q_{1} q_{2}-q_{3}^{2}\right) \gamma \bar{A}_{12}(t)}\right\}=0 .
\end{aligned}
$$


By separating the variables $x, m$, and $m^{2}$, we derive the following ordinary differential equations with associated boundary conditions

$$
\left\{\begin{array}{l}
\bar{A}_{12}^{\prime}(t)+r \bar{A}_{12}(t)=0, \bar{A}_{12}(T)=1, \\
\bar{B}_{12}^{\prime}(t)=0, \bar{B}_{12}(t)=0, \\
\bar{L}_{12}^{\prime}(t)-\frac{q_{4} \sigma_{1} q_{3} A_{12}(t)}{\left(q_{1} q_{2}-q_{3}^{2}\right) \gamma \bar{A}_{12}(t)}-\frac{2 q_{4} q_{9}(t) \bar{B}_{12}(t)}{\left(q_{1} q_{2}-q_{3}^{2}\right) \gamma}=0, \bar{L}_{12}(T)=0, \\
\bar{N}_{12}^{\prime}(t)+\left(c-c_{1}\right) \bar{A}_{12}(t)+\frac{\bar{B}_{12}(t)}{\left(T_{0}-t\right)^{2}}-\frac{q_{4} q_{9}(t) \bar{L}_{12}(t)}{\left(q_{1} q_{2}-q_{3}^{2}\right) \gamma}+\frac{q_{4} q_{8} A_{12}(t)}{\left(q_{1} q_{2}-q_{3}^{2}\right) \gamma \bar{A}_{12}(t)}=0, \bar{N}_{12}(T)=0 .
\end{array}\right.
$$

By solving the above equations ((47) and (49)), we have

$$
\left\{\begin{array}{l}
A_{12}(t)=\bar{A}_{12}(t)=e^{r(T-t)}, \\
\bar{B}_{12}(t)=0, \\
\bar{L}_{12}(t)=-\frac{q_{4} \sigma_{1} q_{3}(T-t)}{\left(q_{1} q_{2}-q_{3}^{2}\right) \gamma}, \\
\bar{N}_{12}(t)=\frac{\left(c-c_{1}\right)\left[e^{r(T-t)}-1\right]}{r}+\frac{q_{4} q_{8}(T-t)}{\left(q_{1} q_{2}-q_{3}^{2}\right) \gamma}-\frac{\sigma_{1} q_{3} q_{4}^{2}\left(\rho \sigma_{2} q_{1}+\sigma_{1} q_{3}\right)}{\gamma\left(q_{1} q_{2}-q_{3}^{2}\right)^{2}} \\
{\left[\begin{array}{l}
\left.T-t+\left(T_{0}-T\right) \ln \frac{T_{0}-T}{T_{0}-t}\right], \\
N_{12}(t)=\frac{\left(c-c_{1}\right)\left[e^{r(T-t)}-1\right]}{r}+\frac{q_{4} q_{8}(T-t)}{\left(q_{1} q_{2}-q_{3}^{2}\right) \gamma}+\frac{\sigma_{1} q_{3} q_{4}^{2}\left(\rho \sigma_{2} q_{1}+\sigma_{1} q_{3}\right)(T-t)}{\gamma\left(q_{1} q_{2}-q_{3}^{2}\right)^{2}} \\
B_{12}(t)=\frac{q_{2}\left(\sigma_{1} q_{3}\right)^{2}(T-t)}{2 \gamma\left(q_{1} q_{2}-q_{3}^{2}\right)^{2}}, \\
L_{12}(t)=-\frac{\sigma_{1} q_{2} q_{3}}{\gamma\left(q_{1} q_{2}-q_{3}^{2}\right)^{2}}\left[\frac{q_{4} \sigma_{1} q_{3}\left(\rho \sigma_{2} q_{1}+\sigma_{1} q_{3}\right)}{q_{1} q_{2}-q_{3}^{2}}-q_{8}\right](T-t)-\frac{\sigma_{1} q_{3} q_{4}\left(\rho \sigma_{1} \sigma_{2} q_{3}\right.}{\gamma\left(q_{1} q_{2}\right.}
\end{array}\right.}
\end{array}\right.
$$

In summary of the above derivation and according to Theorem 3, we derive the following theorem.

Theorem 4. For the postdefault case, i.e., the case $z=1$, the solution to problem (16) is given as follows. The closed-loop equilibrium reinsurance strategy is given by

$$
a^{*}(t)=\frac{q_{6}-q_{7}(t) \bar{L}_{11}(t)-\left[\sigma_{1} q_{2}+2 q_{7}(t) \bar{B}_{11}(t)\right] m}{\left(q_{1} q_{2}-q_{3}^{2}\right) \gamma e^{r(T-t)}} \vee 0
$$

the closed-loop equilibrium investment strategies are given by 


$$
\pi_{1}^{*}(t)= \begin{cases}\frac{q_{8}-q_{9}(t) \bar{L}_{11}(t)-\left[\sigma_{1} q_{3}+2 q_{9}(t) \bar{B}_{11}(t)\right] m}{\left(q_{1} q_{2}-q_{3}^{2}\right) \gamma e^{r(T-t)},} & \text { for } t \in \mathcal{O}_{1}, \\ \frac{q_{8}-q_{9}(t) \bar{L}_{12}(t)-\left[\sigma_{1} q_{3}+2 q_{9}(t) \bar{B}_{12}(t)\right] m}{\left(q_{1} q_{2}-q_{3}^{2}\right) \gamma e^{r(T-t)}}, & \text { for } t \in \mathcal{O}_{2},\end{cases}
$$

and $\pi_{2}^{*}(t)=0$; the corresponding value function is given by

$$
W(t, x, m, 1)= \begin{cases}x e^{r(T-t)}+B_{11}(t) m^{2}+L_{11}(t) m+N_{11}(t), & \text { for } t \in \mathcal{O}_{1}, \\ x e^{r(T-t)}+B_{12}(t) m^{2}+L_{12}(t) m+N_{12}(t), & \text { for } t \in \mathcal{O}_{2},\end{cases}
$$

and the corresponding variance of the terminal wealth is given

by

$$
\operatorname{Var}_{t, x, m, 1}\left(X_{T}^{\pi^{*}}\right)= \begin{cases}\frac{2}{\gamma}\left\{\left[B_{11}-\bar{B}_{11}\right] m^{2}+\left[L_{11}-\bar{L}_{11}\right] m+N_{11}-\bar{N}_{11}\right\}, & \text { for } t \in \mathcal{O}_{1}, \\ \frac{2}{\gamma}\left\{\left[B_{12}-\bar{B}_{12}\right] m^{2}+\left[L_{12}-\bar{L}_{12}\right] m+N_{12}-\bar{N}_{12}\right\}, & \text { for } t \in \mathcal{O}_{2} .\end{cases}
$$

4.2. Predefault Case: $z=0$. In this section, we will address the predefault case, i.e., the case $z=0$. Suppose that there are two functions $W(t, x, m, 0)$ and $g(t, x, m, 0)$ satisfying
Theorem 2. Then, the first equation of equation (23) can be rewritten as

$$
\begin{aligned}
& \sup _{\pi \in \Pi}\left\{W_{t}(t, x, m, 0)+\left[r x+c-c_{1}+a(t)\left(c_{1}+\sigma_{1}\left(\delta_{0}-m\right)\right)+\pi_{1}(t)(\mu-r)+\pi_{2}(t) \delta\right] W_{x}(t, x, m, 0)\right. \\
&+ \frac{1}{2}\left[a^{2}(t) \sigma_{1}^{2}+\pi_{1}^{2}(t) \sigma_{2}^{2}+2 \rho \sigma_{1} \sigma_{2} a(t) \pi_{1}(t)\right]\left[W_{x x}(t, x, m, 0)-\gamma g_{x}^{2}(t, x, m, 0)\right] \\
&+ {\left[\frac{a(t) \sigma_{1}}{T_{0}-t}+\frac{\rho \pi_{1}(t) \sigma_{2}}{T_{0}-t}\right]\left[W_{x m}(t, x, m, 0)-\gamma g_{x}(t, x, m, 0) g_{m}(t, x, m, 0)\right] } \\
&+ \frac{1}{2\left(T_{0}-t\right)^{2}}\left[W_{m m}(t, x, m, 0)-\gamma g_{m}^{2}(t, x, m, 0)\right]+\lambda_{1} E[W(t, x-a(t) Y, m, 0)-W(t, x, m, 0)] \\
&-\frac{\lambda_{1} \gamma}{2} E\left\{[g(t, x-a(t) Y, m, 0)-g(t, x, m, 0)]^{2}\right\}+\lambda_{2} E\left[W\left(t, x+\pi_{1}(t) U, m, 0\right)-W(t, x, m, 0)\right] \\
&-\frac{\lambda_{1} \gamma}{2} E\left\{\left[g\left(t, x-\pi_{1}(t) U, m, 0\right)-g(t, x, m, 0)\right]^{2}\right\}+\lambda E\left[W\left(t, x-a(t) Y+\pi_{1}(t) U, m, 0\right)\right. \\
&-W(t, x, m, 0)]-\frac{\lambda_{1} \gamma}{2} E\left\{\left[g\left(t, x-a(t) Y+\pi_{1}(t), m, 0\right)-g(t, x, m, 0)\right]^{2}\right\} \\
&+ h^{P} E\left[W\left(t, x-\pi_{2}(t) \zeta, m, 1\right)-W(t, x, m, 0)\right] \\
&\left.-\frac{\gamma h^{P}}{2}\left\{\left[g\left(t, x-\pi_{2}(t) \zeta, m, 1\right)-g(t, x, m, 0)\right]^{2}\right\}\right\}=0 .
\end{aligned}
$$

Due to the structure of the second equation of equations (23) and (55) and the boundary conditions $W(T, x, m, 0)=$ $x$ and $g(T, x, m, 0)=x$, we try to guess the solutions to the second equation of (23) and (55) have the following forms:

$$
\left\{\begin{array}{l}
g(t, x, m, 0)=\bar{A}_{2}(t) x+\bar{B}_{2}(t) m^{2}+\bar{L}_{2}(t) m+\bar{N}_{2}(t), \\
W(t, x, m, 0)=A_{2}(t) x+B_{2}(t) m^{2}+L_{2}(t) m+N_{2}(t),
\end{array}\right.
$$

with boundary conditions

$$
\left\{\begin{array}{l}
\bar{A}_{2}(t)=0, \bar{B}_{2}(t)=\bar{L}_{2}(t)=\bar{N}_{2}(t)=0, \\
A_{2}(t)=1, B_{2}(t)=L_{2}(t)=N_{2}(t)=0 .
\end{array}\right.
$$

The corresponding partial derivatives are given by 


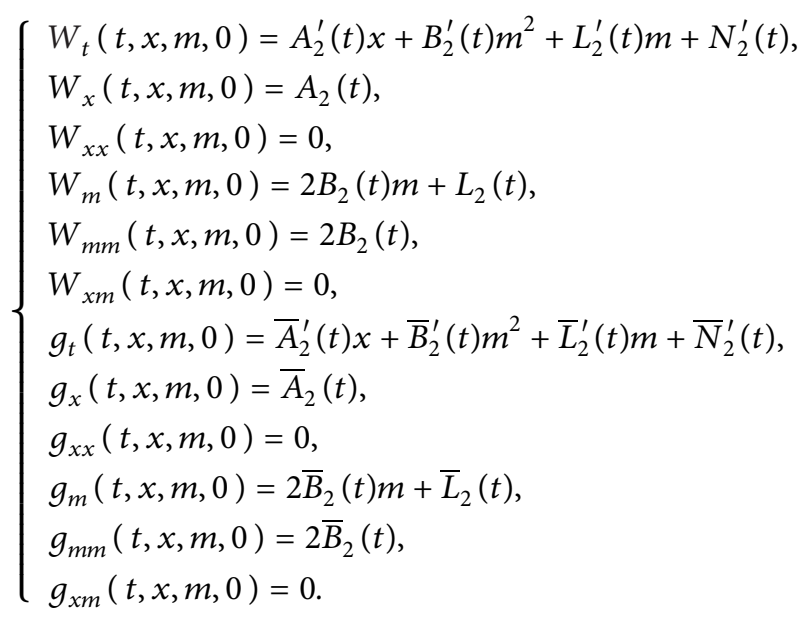

Plugging (56) and the above partial derivatives into (55), we obtain after simplification

$$
\begin{aligned}
& {\left[A_{2}^{\prime}(t)+r A_{2}(t)\right] x+B_{2}^{\prime}(t) m^{2}+L_{2}^{\prime}(t) m} \\
& \quad+N_{2}^{\prime}(t)+\left(c-c_{1}\right) A_{2}(t) \\
& \quad+\frac{1}{2\left(T_{0}-t\right)^{2}}\left[2 B_{2}(t)-\gamma\left(2 \bar{B}_{2}(t) m+\bar{L}_{2}(t)\right)^{2}\right] \\
& \quad+\sup _{\pi \in \Pi} \mathscr{C}\left(a(t), \pi_{1}(t), \pi_{2}(t)\right)=0,
\end{aligned}
$$

$$
\begin{aligned}
\mathscr{C}\left(a(t), \pi_{1}(t), \pi_{2}(t)\right)= & -\frac{\gamma}{2}\left[\sigma_{1}^{2}+\left(\lambda_{1}+\lambda\right) \mu_{12}\right] \bar{A}_{2}^{2}(t) a^{2}(t)+\left\{\left[c_{1}+\sigma_{1}\left(\delta_{0}-m\right)-\left(\lambda_{1}+\lambda\right) \mu_{11}\right] A_{2}(t)\right. \\
& \left.-\frac{a(t) \sigma_{1}}{T_{0}-t}\left[2 \bar{B}_{2}(t) m+\bar{L}_{1}(t)\right] \gamma \bar{A}_{2}(t)\right\} a(t)-\frac{\gamma}{2}\left[\sigma_{2}^{2}+\left(\lambda_{2}+\lambda\right) \mu_{22}\right] \bar{A}_{2}^{2}(t) \pi_{1}^{2}(t) \\
& +\left\{\left[\mu-r+\left(\lambda_{2}+\lambda\right) \mu_{21}\right] A_{2}(t)-\frac{\rho \sigma_{2}}{T_{0}-t}\left[2 \bar{B}_{2}(t) m+\bar{L}_{2}(t)\right] \gamma \bar{A}_{2}(t)\right\} \pi_{1}(t) \\
& +\left(\lambda \mu_{11} \mu_{21}-\rho \sigma_{1} \sigma_{2}\right) \gamma a(t) \pi_{1}(t) \bar{A}_{2}^{2}(t)+\pi_{2}(t) \delta A_{2}(t)+h^{P}\left[\left(A_{1}(t)-A_{2}(t)\right) x+\left(B_{1}(t)-B_{2}(t)\right) m^{2}\right. \\
& \left.+\left(L_{1}(t)-L_{2}(t)\right) m+\left(N_{1}(t)-N_{2}(t)\right)-\pi_{2}(t) \zeta A_{1}(t)\right]-\frac{\gamma h^{P}}{2}\left\{\left[\left(\bar{A}_{1}(t)-\bar{A}_{2}(t)\right) x\right.\right. \\
& \left.\left.+\left(\bar{B}_{1}(t)-\bar{B}_{2}(t)\right) m^{2}+\left(\bar{L}_{1}(t)-\bar{L}_{2}(t)\right) m+\left(\bar{N}_{1}(t)-\bar{N}_{2}(t)\right)-\pi_{2}(t) \zeta \bar{A}_{1}(t)\right]^{2}\right\} .
\end{aligned}
$$

Setting $\left(\partial \mathscr{C}\left(a(t), \pi_{1}(t), \pi_{2}(t)\right) / \partial a(t)\right)=0, \quad(\partial \mathscr{C}(a(t)$, $\left.\left.\pi_{1}(t), \pi_{2}(t)\right) / \partial \pi_{1}(t)\right)=0, \quad$ and $\quad\left(\partial \mathscr{C}\left(a(t), \pi_{1}(t), \pi_{2}(t)\right) /\right.$ $\left.\partial \pi_{2}(t)\right)=0$, we obtain

$$
\left\{\begin{array}{l}
\bar{a}(t)=\frac{q_{6} A_{2}(t)-q_{7}(t) \bar{A}_{2}(t) \bar{L}_{2}(t)-\left[\sigma_{1} q_{2} A_{2}(t)+2 q_{7}(t) \bar{A}_{2}(t) \bar{B}_{2}(t)\right] m}{\left(q_{1} q_{2}-q_{3}^{2}\right) \gamma \bar{A}_{2}^{2}(t)} \\
\bar{\pi}_{1}(t)=\frac{q_{8} A_{2}(t)-q_{9}(t) \bar{A}_{2}(t) \bar{L}_{2}(t)-\left[\sigma_{1} q_{3} A_{2}(t)+2 q_{9}(t) \bar{A}_{2}(t) \bar{B}_{2}(t)\right] m}{\left(q_{1} q_{2}-q_{3}^{2}\right) \gamma \bar{A}_{2}^{2}(t)} \\
\bar{\pi}_{2}(t)=\frac{\delta A_{2}}{\gamma \zeta^{2} h^{P} \bar{A}_{1}^{2}}-\frac{A_{1}(t)}{\gamma \zeta \bar{A}_{1}^{2}}+\frac{1}{\zeta \bar{A}_{1}(t)}\left\{\left[\bar{A}_{1}(t)-\bar{A}_{2}(t)\right] x+\left[\bar{B}_{1}(t)-\bar{B}_{2}(t)\right] m^{2}\right. \\
\left.+\left[\bar{L}_{1}(t)-\bar{L}_{2}(t)\right] m+\bar{N}_{1}(t)-\bar{N}_{2}(t)\right\} .
\end{array}\right.
$$


Similar to Section 4.1, we discuss the closed-loop equilibrium reinsurance strategy in the following two cases.

4.2.1. $\quad \bar{a}(t)>0$. In this section, we assume that $A_{2}(t)=A_{21}(t), \quad B_{2}(t)=B_{21}(t), \quad L_{2}(t)=L_{21}(t), \quad N_{2}(t)=$
$N_{21}(t), \bar{A}_{2}(t)=\bar{A}_{21}(t), \bar{B}_{2}(t)=\bar{B}_{21}(t), \bar{L}_{2}(t)=\bar{L}_{21}(t)$, and $\bar{N}_{2}(t)=\bar{N}_{21}(t)$. Furthermore, from (61), we can obtain the closed-loop equilibrium reinsurance and investment strategies are given by

$$
\left\{\begin{array}{l}
a^{*}(t)=\frac{q_{6} A_{21}(t)-q_{7}(t) \bar{A}_{21}(t) \bar{L}_{21}(t)-\left[\sigma_{1} q_{2} A_{21}(t)+2 q_{7}(t) \bar{A}_{21}(t) \bar{B}_{21}(t)\right] m}{\left(q_{1} q_{2}-q_{3}^{2}\right) \gamma \bar{A}_{21}^{2}(t)} \\
\pi_{1}^{*}(t)=\frac{q_{8} A_{21}(t)-q_{9}(t) \bar{A}_{21}(t) \bar{L}_{21}(t)-\left[\sigma_{1} q_{3} A_{21}(t)+2 q_{9}(t) \bar{A}_{21}(t) \bar{B}_{21}(t)\right] m}{\left(q_{1} q_{2}-q_{3}^{2}\right) \gamma \bar{A}_{2}^{2}(t)} \\
\bar{\pi}_{2}(t)=\frac{\delta A_{21}}{\gamma \zeta^{2} h^{P} \bar{A}_{1}^{2}}-\frac{A_{1}(t)}{\gamma \zeta \bar{A}_{1}^{2}}+\frac{1}{\zeta \bar{A}_{1}(t)}\left\{\left[\bar{A}_{1}(t)-\bar{A}_{21}(t)\right] x+\left[\bar{B}_{1}(t)-\bar{B}_{21}(t)\right] m^{2}\right. \\
\left.+\left[\bar{L}_{1}(t)-\bar{L}_{21}(t)\right] m+\bar{N}_{1}(t)-\bar{N}_{21}(t)\right\}
\end{array}\right.
$$

For presentation convenience, we introduce the following notations:

$$
\begin{aligned}
& \left\{\begin{array}{l}
\mathcal{O}_{3}=\left\{t \mid q_{6}-q_{7}(t) \bar{L}_{21}(t)-\left[\sigma_{1} q_{2}+2 q_{7}(t) \bar{B}_{21}(t)\right] m>0\right\}, \\
\mathcal{O}_{4}=\left\{t \mid q_{6}-q_{7}(t) \bar{L}_{21}(t)-\left[\sigma_{1} q_{2}+2 q_{7}(t) \bar{B}_{21}(t)\right] m \leq 0\right\}, \\
\bar{\Gamma}_{11}(t)=\frac{q_{6} A_{21}(t)-q_{7}(t) \bar{A}_{21}(t) \bar{L}_{21}(t)}{\left(q_{1} q_{2}-q_{3}^{2}\right) \gamma \bar{A}_{21}^{2}(t)} \\
\bar{\Gamma}_{21}(t)=\frac{\sigma_{1} q_{2} A_{21}(t)+2 q_{7}(t) \bar{A}_{21}(t) \bar{B}_{21}(t)}{\left(q_{1} q_{2}-q_{3}^{2}\right) \gamma \bar{A}_{21}^{2}(t)} \\
\bar{\Gamma}_{31}(t)=\frac{q_{8} A_{21}(t)-q_{9}(t) \bar{A}_{21}(t) \bar{L}_{21}(t)}{\left(q_{1} q_{2}-q_{3}^{2}\right) \gamma \bar{A}_{21}^{2}(t)} \\
\bar{\Gamma}_{41}(t)=\frac{\sigma_{1} q_{3} A_{21}(t)+2 q_{9}(t) \bar{A}_{21}(t) \bar{B}_{21}(t)}{\left(q_{1} q_{2}-q_{3}^{2}\right) \gamma \bar{A}_{21}^{2}(t)} \\
\bar{\Gamma}_{51}(t)=q_{4} A_{21}(t)-\frac{\rho \sigma_{2} \gamma}{T_{0}-t} \bar{A}_{21}(t) \bar{L}_{21}(t), \\
\bar{\Gamma}_{61}(t)=\frac{2 \rho \sigma_{2} \gamma}{T_{0}-t} \bar{A}_{21}(t) \bar{B}_{21}(t) \\
\bar{\Gamma}_{71}(t)=q_{5} A_{21}(t) \\
\bar{\Gamma}_{81}(t)=\sigma_{1} A_{21}(t)+\frac{2 \sigma_{1} \gamma}{T_{0}-t} \bar{A}_{21}(t) \bar{B}_{21}(t) .
\end{array}\right.
\end{aligned}
$$


Plugging (62) into (59), we obtain after simplification

$$
\begin{aligned}
& \left\{A_{21}^{\prime}(t)+\left[r+\frac{\delta}{\zeta}-\frac{\delta}{\zeta} \bar{A}_{21}(t) e^{-r(T-t)}-h^{P}\right] A_{21}(t)+h^{P} \bar{A}_{21}(t)\right\} x \\
& +\left\{B_{21}^{\prime}(t)-\frac{2 \gamma \bar{B}_{21}^{2}(t)}{\left(T_{0}-t\right)^{2}}-\frac{\gamma q_{2}}{2} \bar{A}_{21}^{2}(t) \bar{\Gamma}_{41}^{2}(t)-\frac{\gamma q_{1}}{2} \bar{A}_{21}^{2}(t) \bar{\Gamma}_{21}^{2}(t)+\gamma q_{5} \bar{A}_{21}^{2}(t) \bar{\Gamma}_{21}(t) \bar{\Gamma}_{41}(t)+\bar{\Gamma}_{21}(t) \bar{\Gamma}_{81}(t)\right. \\
& \left.+\bar{\Gamma}_{41}(t) \bar{\Gamma}_{61}(t)+\frac{\delta}{\zeta} A_{21}(t)\left[\bar{B}_{1}(t)-\bar{B}_{21}(t)\right] e^{-r(T-t)}+h^{P}\left[B_{1}(t)-\bar{B}_{1}(t)-B_{21}(t)+\bar{B}_{21}(t)\right]\right\} m^{2} \\
& +\left\{\frac{L_{21}^{\prime}(t)-2 \gamma \bar{B}_{21}(t) \bar{L}_{21}(t)}{\left(T_{0}-t\right)^{2}-\frac{\gamma q_{2}}{2} \bar{A}_{21}^{2}(t) \bar{\Gamma}_{31}(t) \bar{\Gamma}_{41}(t)-\frac{\gamma q_{1}}{2} \bar{A}_{21}^{2}(t) \bar{\Gamma}_{11}(t) \bar{\Gamma}_{21}(t)}\right. \\
& -\gamma q_{5} \bar{A}_{21}^{2}(t)\left[\bar{\Gamma}_{21}(t) \bar{\Gamma}_{31}(t)+\bar{\Gamma}_{11}(t) \bar{\Gamma}_{41}(t)\right]-\bar{\Gamma}_{11}(t) \bar{\Gamma}_{81}(t)-\bar{\Gamma}_{31}(t) \bar{\Gamma}_{61}(t)+\frac{\delta}{\zeta} A_{21}(t)\left[\bar{L}_{1}(t)\right. \\
& \left.\left.-\bar{L}_{21}(t)\right] e^{-r(T-t)}+h^{P}\left[L_{1}(t)-\bar{L}_{1}(t)-L_{21}(t)+\bar{L}_{21}(t)\right]-\bar{\Gamma}_{41}(t) \bar{\Gamma}_{51}(t)-\bar{\Gamma}_{21}(t) \bar{\Gamma}_{71}(t)\right\} m \\
& +\left\{N_{21}^{\prime}(t)+\left(c-c_{1}\right) A_{21}(t)+\frac{B_{21}(t)}{\left(T_{0}-t\right)^{2}}-\frac{\gamma \bar{L}_{1}^{2}}{2\left(T_{0}-t\right)^{2}}-\frac{\gamma q_{2}}{2} \bar{A}_{21}^{2}(t) \bar{\Gamma}_{31}^{2}(t)-\frac{\gamma q_{1}}{2} \bar{A}_{21}^{2}(t) \bar{\Gamma}_{11}^{2}(t)\right. \\
& +\gamma q_{5} \bar{A}_{21}^{2}(t) \bar{\Gamma}_{11}(t) \bar{\Gamma}_{31}(t)+\bar{\Gamma}_{11}(t) \bar{\Gamma}_{71}(t)+\bar{\Gamma}_{31}(t) \bar{\Gamma}_{51}(t)+\frac{\delta^{2}}{\gamma \zeta^{2} h^{P}} \frac{A_{21}^{2}(t)}{\bar{A}_{1}^{2}(t)}-\frac{2 \delta}{\gamma \zeta} \frac{A_{21}(t)}{\bar{A}_{1}(t)} \\
& \left.+\frac{\delta}{\zeta} \frac{A_{21}(t)}{\bar{A}_{1}(t)}\left[\bar{N}_{1}(t)-\bar{N}_{21}(t)\right]+h^{P}\left[N_{1}(t)-\bar{N}_{1}(t)-N_{21}(t)+\bar{N}_{21}(t)\right]-\frac{h^{P}}{\gamma}-\frac{\gamma\left(h^{P}\right)^{2}}{2}\left[\frac{1}{\gamma}-\frac{\delta A_{21}(t)}{\gamma \zeta h^{P} \bar{A}_{1}(t)}\right]^{2}\right\}=0
\end{aligned}
$$


Thus, we need to solve the following ordinary differential equations with associated boundary conditions

$$
\begin{aligned}
& \int A_{21}^{\prime}(t)+\left[r+\frac{\delta}{\zeta}-\frac{\delta}{\zeta} \bar{A}_{21}(t) e^{-r(T-t)}-h^{P}\right] A_{21}(t)+h^{P} \bar{A}_{21}(t)=0, \\
& A_{21}(T)=1 \\
& B_{21}^{\prime}(t)-h^{P} B_{21}(t)-\frac{2 \gamma \bar{B}_{21}^{2}(t)}{\left(T_{0}-t\right)^{2}}-\frac{\gamma q_{2}}{2} \bar{A}_{21}^{2}(t) \bar{\Gamma}_{41}^{2}(t)-\frac{\gamma q_{1}}{2} \bar{A}_{21}^{2}(t) \bar{\Gamma}_{21}^{2}(t)+\gamma q_{5} \bar{A}_{21}^{2}(t) \bar{\Gamma}_{21}(t) \bar{\Gamma}_{41}(t) \\
& +\bar{\Gamma}_{21}(t) \bar{\Gamma}_{81}(t)+\bar{\Gamma}_{41}(t) \bar{\Gamma}_{61}(t)+\frac{\delta}{\zeta} A_{21}(t)\left[\bar{B}_{1}(t)-\bar{B}_{21}(t)\right] e^{-r(T-t)} \\
& +h^{P}\left[B_{1}(t)-\bar{B}_{1}(t)+\bar{B}_{21}(t)\right]=0 \\
& B_{21}(T)=0 \\
& L_{21}^{\prime}(t)-h^{P} L_{21}(t)-\frac{2 \gamma \bar{B}_{21}(t) \bar{L}_{21}(t)}{\left(T_{0}-t\right)^{2}}-\frac{\gamma q_{2}}{2} \bar{A}_{21}^{2}(t) \bar{\Gamma}_{31}(t) \bar{\Gamma}_{41}(t)-\frac{\gamma q_{1}}{2} \bar{A}_{21}^{2}(t) \bar{\Gamma}_{11}(t) \bar{\Gamma}_{21}(t) \\
& -\gamma q_{5} \bar{A}_{21}^{2}(t)\left[\bar{\Gamma}_{21}(t) \bar{\Gamma}_{31}(t)+\bar{\Gamma}_{11}(t) \bar{\Gamma}_{41}(t)\right]-\bar{\Gamma}_{11}(t) \bar{\Gamma}_{81}(t)-\bar{\Gamma}_{31}(t) \bar{\Gamma}_{61}(t)-\bar{\Gamma}_{41}(t) \bar{\Gamma}_{51}(t) \\
& -\bar{\Gamma}_{21}(t) \bar{\Gamma}_{71}(t)+\frac{\delta}{\zeta} A_{21}(t)\left[\bar{L}_{1}(t)-\bar{L}_{21}(t)\right] e^{-r(T-t)}+h^{P}\left[L_{1}(t)-\bar{L}_{1}(t)+\bar{L}_{21}(t)\right]=0, \\
& L_{21}(T)=0 \\
& N_{21}^{\prime}(t)-h^{P} N_{21}(t)+\left(c-c_{1}\right) A_{21}(t)+\frac{B_{21}(t)}{\left(T_{0}-t\right)^{2}}-\frac{\gamma \bar{L}_{1}^{2}}{2\left(T_{0}-t\right)^{2}}-\frac{\gamma q_{2}}{2} \bar{A}_{21}^{2}(t) \bar{\Gamma}_{31}^{2}(t)-\frac{\gamma q_{1}}{2} \bar{A}_{21}^{2}(t) \bar{\Gamma}_{11}^{2}(t) \\
& +\gamma q_{5} \bar{A}_{21}^{2}(t) \bar{\Gamma}_{11}(t) \bar{\Gamma}_{31}(t)+\bar{\Gamma}_{11}(t) \bar{\Gamma}_{71}(t)+\bar{\Gamma}_{31}(t) \bar{\Gamma}_{51}(t)+\frac{\delta^{2}}{\gamma \zeta^{2} h^{P}} \frac{A_{21}^{2}(t)}{\bar{A}_{1}^{2}(t)}-\frac{2 \delta}{\gamma \zeta} \frac{A_{21}(t)}{\bar{A}_{1}(t)}+\frac{\delta}{\zeta} \frac{A_{21}(t)}{\bar{A}_{1}(t)}\left[\bar{N}_{1}(t)-\bar{N}_{21}(t)\right] \\
& +h^{P}\left[N_{1}(t)-\bar{N}_{1}(t)+\bar{N}_{21}(t)\right]-\frac{h^{P}}{\gamma}-\frac{\gamma\left(h^{P}\right)^{2}}{2}\left[\frac{1}{\gamma}-\frac{\delta A_{21}(t)}{\gamma \zeta h^{P} \bar{A}_{1}(t)}\right]^{2}=0 \\
& N_{21}(T)=0 .
\end{aligned}
$$


Plugging (62) into the second equation of equation (23), we obtain after simplification

$$
\begin{aligned}
& \left\{\bar{A}_{21}^{\prime}(t)+\left[r+\frac{\delta}{\zeta}-\frac{\delta}{\zeta} \bar{A}_{21}(t) e^{-r(T-t)}\right] \bar{A}_{21}(t)\right\} x \\
& +\left\{\frac{\bar{B}_{21}^{\prime}(t)+\left[\frac{2 \sigma_{1} q_{7}(t)}{\left(q_{1} q_{2}-q_{3}^{2}\right) \gamma}-\frac{\delta \bar{A}_{21}(t)}{\zeta \bar{A}_{1}(t)}\right] \bar{B}_{21}(t)+\frac{\sigma_{1}^{2} q_{2} A_{21}(t)}{\left(q_{1} q_{2}-q_{3}^{2}\right) \gamma \bar{A}_{21}(t)}+\delta \bar{A}_{21}(t) \bar{B}_{1}(t)}{\zeta \bar{A}_{1}(t)}\right\} m^{2} \\
& +\left\{\bar{L}_{21}^{\prime}(t)+\left[\frac{\sigma_{1} q_{7}(t)}{\left(q_{1} q_{2}-q_{3}^{2}\right) \gamma}-\frac{\delta \bar{A}_{21}(t)}{\zeta \bar{A}_{1}(t)}\right] \bar{L}_{21}(t)-\frac{\left(\sigma_{1} q_{6}+q_{4} \sigma_{1} q_{3}+q_{5} \sigma_{1} q_{2}\right) A_{21}(t)}{\left(q_{1} q_{2}-q_{3}^{2}\right) \gamma \bar{A}_{21}(t)}\right. \\
& \left.-\frac{\left[2 q_{4} q_{9}(t)+2 q_{5} q_{7}(t)\right] \bar{B}_{21}(t)}{\left(q_{1} q_{2}-q_{3}^{2}\right) \gamma}+\frac{\delta \bar{A}_{21}(t) \bar{L}_{1}(t)}{\zeta \bar{A}_{1}(t)}\right\} m \\
& +\left\{\bar{N}_{21}^{\prime}(t)-\frac{\delta \bar{A}_{21}(t)}{\zeta \bar{A}_{1}(t)} \bar{N}_{21}(t)+\left(c-c_{1}\right) \bar{A}_{1}(t)+\frac{\bar{B}_{21}(t)}{\left(T_{0}-t\right)^{2}}-\frac{\left[q_{4} q_{9}(t)+q_{5} q_{7}(t)\right] \bar{L}_{21}(t)}{\left(q_{1} q_{2}-q_{3}^{2}\right) \gamma}\right. \\
& \left.+\frac{\left(q_{4} q_{8}+q_{5} q_{6}\right) A_{21}(t)}{\left(q_{1} q_{2}-q_{3}^{2}\right) \gamma \bar{A}_{21}(t)}+\frac{\delta^{2} A_{21}(t) \bar{A}_{21}(t)}{\gamma \zeta^{2} h^{P} \bar{A}_{1}(t)}-\frac{\delta \bar{A}_{21}(t)}{\gamma \zeta \bar{A}_{1}(t)}+\frac{\delta \bar{A}_{21}(t)}{\zeta \bar{A}_{1}(t)} \bar{N}_{1}(t)-\frac{\delta A_{21}(t)}{\gamma \zeta \bar{A}_{1}(t)}+\frac{h^{P}}{\gamma}\right\}=0 .
\end{aligned}
$$

Thus, we need to solve the following ordinary differential equations with associated boundary conditions

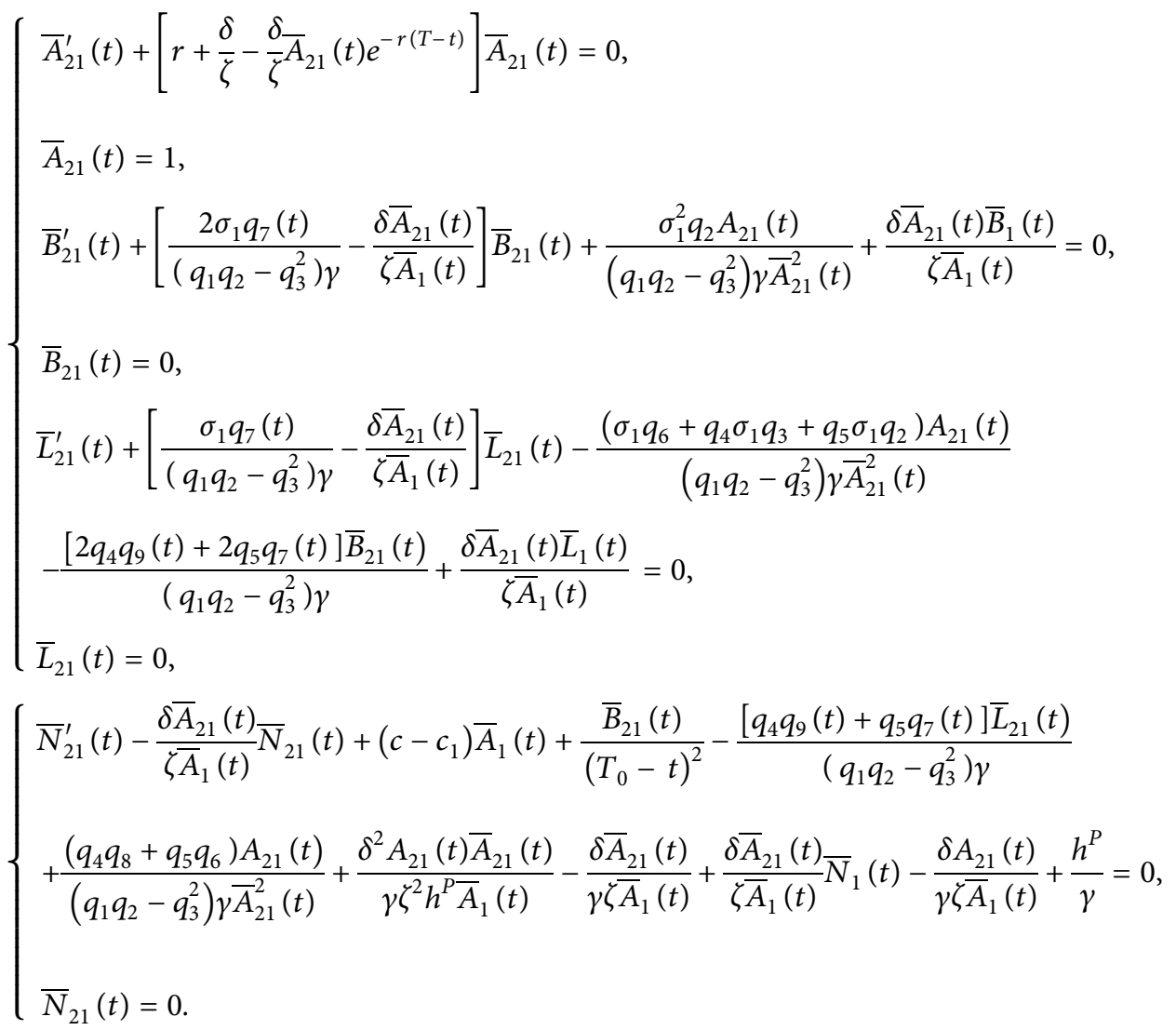


By solving the above equations ((65) and (67)), we have

$$
\begin{aligned}
& \left\{\begin{array}{l}
A_{21}(t)=\bar{A}_{21}(t)=e^{r(T-t)}, \\
\bar{B}_{21}(t)=\left(\frac{T_{0}-t}{T_{0}-T}\right)^{\left(2 \sigma_{1}^{2} q_{2}+2 \rho \sigma_{1} \sigma_{2} q_{3} / q_{1} q_{2}-q_{3}^{2}\right)} e^{-(\delta / \zeta)(T-t)} \\
\times\left\{\int_{t}^{T}\left[\frac{\sigma_{1}^{2} q_{2}}{\left(q_{1} q_{2}-q_{3}^{2}\right) \gamma}+\frac{\delta \bar{B}_{1}(s)}{\zeta}\right]\left(\frac{T_{0}-T}{T_{0}-s}\right)^{\left(2 \sigma_{1}^{2} q_{2}+2 \rho \sigma_{1} \sigma_{2} q_{3} / q_{1} q_{2}-q_{3}^{2}\right)} e^{(\delta / \zeta)(T-s)} \mathrm{d}\right\}, \\
\bar{L}_{21}(t)=\left(\frac{T_{0}-t}{T_{0}-T}\right){ }^{\left(\sigma_{1}^{2} q_{2}+\rho \sigma_{1} \sigma_{2} q_{3} / q_{1} q_{2}-q_{3}^{2}\right)} e^{-(\delta / \zeta)(T-t)}\left\{\int _ { t } ^ { T } \left[\frac{\delta \bar{L}_{1}(s)}{\zeta}-\frac{\left(\sigma_{1} q_{6}+q_{4} \sigma_{1} q_{3}+q_{5} \sigma_{1} q_{2}\right)}{\left(q_{1} q_{2}-q_{3}^{2}\right) \gamma}\right.\right. \\
\left.-\frac{\left[2 q_{4} q_{9}(s)+2 q_{5} q_{7}(s)\right] \bar{B}_{21}(s)}{\left(q_{1} q_{2}-q_{3}^{2}\right) \gamma}\left(\frac{T_{0}-T}{T_{0}-s}\right){ }^{\left(\sigma_{1}^{2} q_{2}+\rho \sigma_{1} \sigma_{2} q_{3} / q_{1} q_{2}-q_{3}^{2}\right)} e^{(\delta / \zeta)(T-s)} \mathrm{d} s\right\}, \\
\bar{N}_{21}(t)=e^{-(\delta / \zeta)(T-t)}\left\{\int _ { t } ^ { T } \left[\left(c-c_{1}\right) e^{r(T-v)}+\frac{\bar{B}_{21}(v)}{\left(T_{0}-v\right)^{2}}-\frac{\left[q_{4} q_{9}(v)+q_{5} q_{7}(v)\right] \bar{L}_{21}(v)}{\left(q_{1} q_{2}-q_{3}^{2}\right) \gamma}\right.\right. \\
\left.\left.+\frac{\left(q_{4} q_{8}+q_{5} q_{6}\right)}{\left(q_{1} q_{2}-q_{3}^{2}\right) \gamma}+\frac{\delta^{2} e^{r(T-v)}}{\gamma \zeta^{2} h^{P}}-\frac{\delta}{\gamma \zeta}+\frac{\delta}{\zeta} \bar{N}_{1}(v)-\frac{\delta}{\gamma \zeta}+\frac{h^{P}}{\gamma}\right] e^{(\delta / \zeta)(T-v)} \mathrm{d} v\right\},
\end{array}\right. \\
& \int B_{21}(t)=e^{-h^{P}(T-t)}\left\{\int _ { t } ^ { T } \left\{-\frac{2 \gamma \bar{B}_{21}^{2}(s)}{\left(T_{0}-s\right)^{2}}-\frac{\gamma q_{2}}{2} \bar{A}_{21}^{2}(s) \bar{\Gamma}_{41}^{2}(s)-\frac{\gamma q_{1}}{2} \bar{A}_{21}^{2}(s) \bar{\Gamma}_{21}^{2}(s)\right.\right. \\
& +\gamma q_{5} \bar{A}_{21}^{2}(s) \bar{\Gamma}_{21}(s) \bar{\Gamma}_{41}(s)+\bar{\Gamma}_{21}(s) \bar{\Gamma}_{81}(s)+\bar{\Gamma}_{41}(s) \bar{\Gamma}_{61}(s)+\frac{\delta}{\zeta}\left[\bar{B}_{1}(s)-\bar{B}_{21}(s)\right] \\
& \left.\left.+h^{P}\left[B_{1}(s)-\bar{B}_{1}(s)+\bar{B}_{21}(s)\right]\right\} e^{h^{P}(T-s)} \mathrm{d} s\right\} \\
& L_{21}(t)=e^{-h^{P}(T-t)}\left\{\int _ { t } ^ { T } \left\{\frac{-2 \gamma \bar{B}_{21}(s) \bar{L}_{21}(s)}{\left(T_{0}-s\right)^{2}-\frac{\gamma q_{2}}{2} \bar{A}_{21}^{2}(s) \bar{\Gamma}_{31}(s) \bar{\Gamma}_{41}(s)-\frac{\gamma q_{1}}{2} \bar{A}_{21}^{2}(s) \bar{\Gamma}_{11}(s) \bar{\Gamma}_{21}(s)}\right.\right. \\
& -\gamma q_{5} \bar{A}_{21}^{2}(s)\left[\bar{\Gamma}_{21}(s) \bar{\Gamma}_{31}(s)+\bar{\Gamma}_{11}(s) \bar{\Gamma}_{41}(s)\right]-\bar{\Gamma}_{11}(s) \bar{\Gamma}_{81}(s)-\bar{\Gamma}_{31}(s) \bar{\Gamma}_{61}(s)-\bar{\Gamma}_{41}(s) \bar{\Gamma}_{51}(s) \\
& \left.\left.-\bar{\Gamma}_{21}(s) \bar{\Gamma}_{71}(s)+\frac{\delta}{\zeta}\left[\bar{L}_{1}(s)-\bar{L}_{21}(s)\right]+h^{P}\left[L_{1}(s)-\bar{L}_{1}(s)+\bar{L}_{21}(s)\right]\right\} e^{h^{P}(T-s)} \mathrm{d} s\right\}, \\
& N_{21}(t)=e^{-h^{P}(T-t)}\left\{\int _ { t } ^ { T } \left\{\left(c-c_{1}\right) e^{r(T-s)}+\frac{B_{21}(s)}{\left(T_{0}-s\right)^{2}}-\frac{\gamma \bar{L}_{1}^{2}}{2\left(T_{0}-s\right)^{2}}-\frac{\gamma q_{2}}{2} e^{2 r(T-s)} \bar{\Gamma}_{31}^{2}(s)\right.\right. \\
& -\frac{\gamma q_{1}}{2} e^{2 r(T-s)} \bar{\Gamma}_{11}^{2}(t)+\gamma q_{5} e^{2 r(T-s)} \bar{\Gamma}_{11}(s) \bar{\Gamma}_{31}(s)+\bar{\Gamma}_{11}(s) \bar{\Gamma}_{71}(s)+\bar{\Gamma}_{31}(s) \bar{\Gamma}_{51}(s) \\
& +\frac{\delta^{2}}{\gamma \zeta^{2} h^{P}}-\frac{2 \delta}{\gamma \zeta}+\frac{\delta}{\zeta}\left[\bar{N}_{1}(s)-\bar{N}_{21}(s)\right]+h^{P}\left[N_{1}(s)-\bar{N}_{1}(s)+\bar{N}_{21}(s)\right]-\frac{h^{P}}{\gamma} \\
& \left.\left.-\frac{\gamma\left(h^{P}\right)^{2}}{2}\left[\frac{1}{\gamma}-\frac{\delta}{\gamma \zeta h^{P}}\right]^{2}\right\} e^{h^{P}(T-s)} \mathrm{d} s\right\}
\end{aligned}
$$


4.2.2. $\bar{a} \leq 0$. In this section, we assume that $A_{2}(t)=A_{22}(t)$, $B_{2}(t)=B_{22}(t), \quad L_{2}(t)=L_{22}(t), \quad N_{2}(t)=N_{22}(t), \quad \bar{A}_{2}(t)=$ $\bar{A}_{22}(t), \bar{B}_{2}(t)=\bar{B}_{22}(t), \bar{L}_{2}(t)=\bar{L}_{22}(t)$, and $\bar{N}_{2}(t)=\bar{N}_{22}(t)$.
For presentation convenience, we introduce the following notations:

$$
\left\{\begin{array}{l}
\bar{\Gamma}_{12}(t)=\frac{q_{6} A_{22}(t)-q_{7}(t) \bar{A}_{22}(t) \bar{L}_{21}(t)}{\left(q_{1} q_{2}-q_{3}^{2}\right) \gamma \bar{A}_{22}^{2}(t)}, \\
\bar{\Gamma}_{22}(t)=\frac{\sigma_{1} q_{2} A_{22}(t)+2 q_{7}(t) \bar{A}_{22}(t) \bar{B}_{22}(t)}{\left(q_{1} q_{2}-q_{3}^{2}\right) \gamma \bar{A}_{22}^{2}(t)} \\
\bar{\Gamma}_{32}(t)=\frac{q_{8} A_{22}(t)-q_{9}(t) \bar{A}_{22}(t) \bar{L}_{22}(t)}{\left(q_{1} q_{2}-q_{3}^{2}\right) \gamma \bar{A}_{22}^{2}(t)} \\
\bar{\Gamma}_{42}(t)=\frac{\sigma_{1} q_{3} A_{22}(t)+2 q_{9}(t) \bar{A}_{22}(t) \bar{B}_{22}(t)}{\left(q_{1} q_{2}-q_{3}^{2}\right) \gamma \bar{A}_{22}^{2}(t)} \\
\bar{\Gamma}_{82}(t)=\sigma_{1} A_{22}(t)+\frac{2 \sigma_{1} \gamma}{T_{0}-t} \bar{A}_{22}(t) \bar{B}_{22}(t) \\
\bar{\Gamma}_{62}(t)=\frac{2 \rho \sigma_{2} \gamma}{T_{0}-t} \bar{A}_{22}(t) \bar{B}_{22}(t) \\
\bar{\Gamma}_{52}(t)=q_{4} A_{22}(t)-\frac{\rho \sigma_{2} \gamma}{T_{0}-t} \bar{A}_{22}(t) \bar{L}_{22}(t),
\end{array}\right.
$$

Since $\bar{a} \leq 0$, we obtain the closed-loop equilibrium reinsurance strategy is $a^{*}(t)=0$. From (61), the closed-loop equilibrium investment strategies $\pi_{1}^{*}(t)$ and $\pi_{2}^{*}(t)$ are, respectively, given by

$$
\left\{\begin{array}{l}
\pi_{1}^{*}(t)=\frac{q_{8} A_{22}(t)-q_{9}(t) \bar{A}_{22}(t) \bar{L}_{22}(t)-\left[\sigma_{1} q_{3} A_{22}(t)+2 q_{9}(t) \bar{A}_{22}(t) \bar{B}_{22}(t)\right] m}{\left(q_{1} q_{2}-q_{3}^{2}\right) \gamma \bar{A}_{22}^{2}(t)}, \\
\bar{\pi}_{2}(t)=\frac{\delta A_{21}}{\gamma \zeta^{2} h^{P} \bar{A}_{1}^{2}}-\frac{A_{1}(t)}{\gamma \zeta \bar{A}_{1}^{2}}+\frac{1}{\zeta \bar{A}_{1}(t)}\left\{\left[\bar{A}_{1}(t)-\bar{A}_{22}(t)\right] x+\left[\bar{B}_{1}(t)-\bar{B}_{22}(t)\right] m^{2}+\left[\bar{L}_{1}(t)-\bar{L}_{22}(t)\right] m+\bar{N}_{1}(t)-\bar{N}_{22}(t)\right\}
\end{array}\right.
$$


Plugging $a^{*}(t)=0$ and (70) into (59) and the second equation equation (23), we obtain after simplification

$$
\begin{aligned}
& \left\{A_{22}^{\prime}(t)+\left[r+\frac{\delta}{\zeta}-\frac{\delta}{\zeta} \bar{A}_{22}(t) e^{-r(T-t)}-h^{P}\right] A_{22}(t)+h^{P} \bar{A}_{22}(t)\right\} x \\
& +\left\{B_{22}^{\prime}(t)-\frac{2 \gamma \bar{B}_{22}^{2}(t)}{\left(T_{0}-t\right)^{2}}-\frac{\gamma q_{2}}{2} \bar{A}_{22}^{2}(t) \bar{\Gamma}_{42}^{2}(t)+\bar{\Gamma}_{42}(t) \bar{\Gamma}_{62}(t)+\frac{\delta}{\zeta} A_{22}(t)\left[\bar{B}_{1}(t)-\bar{B}_{22}(t)\right] e^{-r(T-t)}+h^{P}\left[B_{1}(t)-\bar{B}_{1}(t)-B_{22}(t)+\bar{B}_{22}(t)\right]\right\} m^{2} \\
& +\left\{\frac{L_{22}^{\prime}(t)-2 \gamma \bar{B}_{22}(t) \bar{L}_{22}(t)}{\left(T_{0}-t\right)^{2}-\frac{\gamma q_{2}}{2} \bar{A}_{22}^{2}(t) \bar{\Gamma}_{32}(t) \bar{\Gamma}_{42}(t)-\bar{\Gamma}_{32}(t) \bar{\Gamma}_{62}(t)-\bar{\Gamma}_{42}(t) \bar{\Gamma}_{52}(t)+\frac{\delta}{\zeta} A_{22}(t)\left[\bar{L}_{1}(t)-\bar{L}_{22}(t)\right] e^{-r(T-t)}+h^{P}\left[L_{1}(t)-\bar{L}_{1}(t)-L_{22}(t)+\bar{L}_{22}(t)\right]}\right\} m \\
& +\left\{N_{22}^{\prime}(t)+\left(c-c_{1}\right) A_{22}(t)+\frac{B_{22}(t)}{\left(T_{0}-t\right)^{2}}-\frac{\gamma \bar{L}_{1}^{2}}{2\left(T_{0}-t\right)^{2}}-\frac{\gamma q_{2}}{2} \bar{A}_{22}^{2}(t) \bar{\Gamma}_{32}^{2}(t)+\bar{\Gamma}_{32}(t) \bar{\Gamma}_{52}(t)+\frac{\delta^{2}}{\gamma \zeta^{2} h^{P}} \frac{A_{22}^{2}(t)}{\bar{A}_{1}^{2}(t)}-\frac{2 \delta}{\gamma \zeta} \frac{A_{22}(t)}{\bar{A}_{1}(t)}+\frac{\delta}{\zeta} \frac{A_{22}(t)}{\bar{A}_{1}(t)}\left[\bar{N}_{1}(t)-\bar{N}_{22}(t)\right]\right. \\
& \left.+h^{P}\left[N_{1}(t)-\bar{N}_{1}(t)-N_{22}(t)+\bar{N}_{22}(t)\right]-\frac{h^{P}}{\gamma}-\frac{\gamma\left(h^{P}\right)^{2}}{2}\left[\frac{1}{\gamma}-\frac{\delta A_{22}(t)}{\gamma \zeta h^{P} \bar{A}_{1}(t)}\right]^{2}\right\}=0 \\
& \left\{\bar{A}_{22}^{\prime}(t)+\left[r+\frac{\delta}{\zeta}-\frac{\delta}{\zeta} \bar{A}_{22}(t) e^{-r(T-t)}\right] \bar{A}_{22}(t)\right\} x \\
& +\left\{\frac{\bar{B}_{22}^{\prime}(t)-\frac{\delta \bar{A}_{22}(t)}{\zeta \bar{A}_{1}(t)} \bar{B}_{22}(t)+\delta \bar{A}_{22}(t) \bar{B}_{1}(t)}{\zeta \bar{A}_{1}(t)}\right\} m^{2} \\
& +\left\{\frac{\bar{L}_{22}^{\prime}(t)-\frac{\delta \bar{A}_{22}(t)}{\zeta \bar{A}_{1}(t)} \bar{L}_{22}(t)-\frac{q_{4} \sigma_{1} q_{3}}{\left(q_{1} q_{2}-q_{3}^{2}\right) \gamma \bar{A}_{22}(t)}-\frac{2 q_{4} q_{9}(t) \bar{B}_{22}(t)}{\left(q_{1} q_{2}-q_{3}^{2}\right) \gamma}+\delta \bar{A}_{22}(t) \bar{L}_{1}(t)}{\zeta \bar{A}_{1}(t)}\right\} m \\
& \left\{\bar{N}_{22}^{\prime}(t)-\frac{\delta \bar{A}_{22}(t)}{\zeta \bar{A}_{1}(t)} \bar{N}_{22}(t)+\left(c-c_{1}\right) \bar{A}_{1}(t)+\frac{\bar{B}_{22}(t)}{\left(T_{0}-t\right)^{2}}-\frac{q_{4} q_{9}(t) \bar{L}_{22}(t)}{\left(q_{1} q_{2}-q_{3}^{2}\right) \gamma}+\frac{q_{4} q_{8} A_{22}(t)}{\left(q_{1} q_{2}-q_{3}^{2}\right) \gamma \bar{A}_{22}(t)}+\frac{\delta^{2} A_{22}(t) \bar{A}_{22}(t)}{\gamma \zeta^{2} h^{P} \bar{A}_{1}(t)}\right. \\
& \left.-\frac{\delta \bar{A}_{22}(t)}{\gamma \zeta \bar{A}_{1}(t)}+\frac{\delta \bar{A}_{22}(t)}{\zeta \bar{A}_{1}(t)} \bar{N}_{1}(t)-\frac{\delta A_{22}(t)}{\gamma \zeta \bar{A}_{1}(t)}+\frac{h^{P}}{\gamma}\right\}=0 .
\end{aligned}
$$

Thus, we need to solve the following ordinary differential equations with associated boundary conditions 


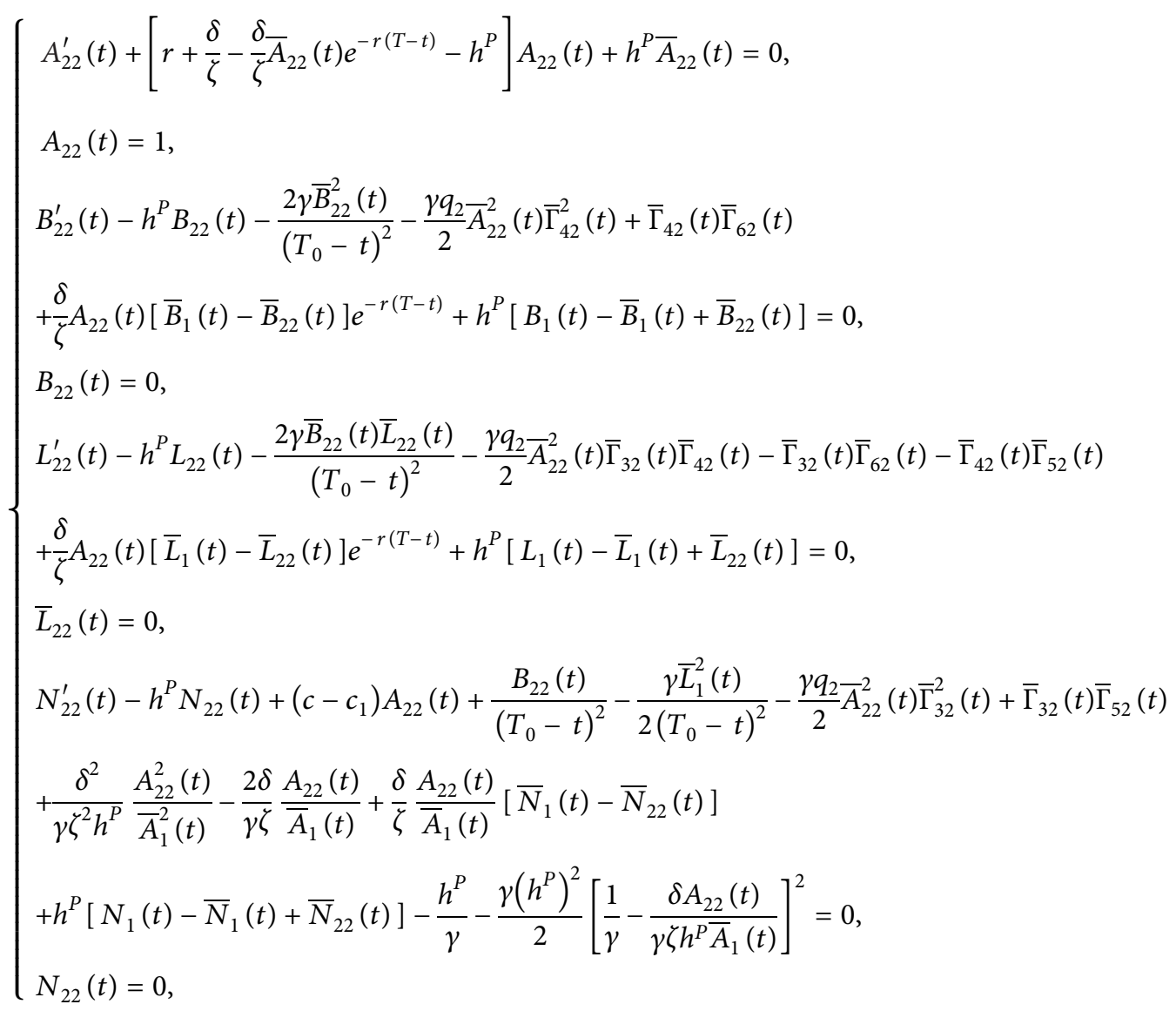

and

$$
\begin{aligned}
& \int \bar{A}_{22}^{\prime}(t)+\left[r+\frac{\delta}{\zeta}-\frac{\delta}{\zeta} \bar{A}_{22}(t) e^{-r(T-t)}\right] \bar{A}_{22}(t)=0 \\
& \bar{A}_{22}(t)=1 \text {, } \\
& \bar{B}_{22}^{\prime}(t)-\frac{\delta \bar{A}_{22}(t)}{\zeta \bar{A}_{1}(t)} \bar{B}_{22}(t)+\frac{\delta \bar{A}_{22}(t) \bar{B}_{1}(t)}{\zeta \bar{A}_{1}(t)}=0, \\
& \bar{B}_{22}(t)=0 \text {, } \\
& \left\{\bar{L}_{22}^{\prime}(t)-\frac{\delta \bar{A}_{22}(t)}{\zeta \bar{A}_{1}(t)} \bar{L}_{22}(t)-\frac{q_{4} \sigma_{1} q_{3}}{\left(q_{1} q_{2}-q_{3}^{2}\right) \gamma \bar{A}_{22}^{2}(t)}-\frac{2 q_{4} q_{9}(t) \bar{B}_{22}(t)}{\left(q_{1} q_{2}-q_{3}^{2}\right) \gamma}+\frac{\delta \bar{A}_{22}(t) \bar{L}_{1}(t)}{\zeta \bar{A}_{1}(t)}=0,\right. \\
& \bar{L}_{22}(t)=0 \\
& \bar{N}_{22}^{\prime}(t)-\frac{\delta \bar{A}_{22}(t)}{\zeta \bar{A}_{1}(t)} \bar{N}_{22}(t)+\left(c-c_{1}\right) \bar{A}_{1}(t)+\frac{\bar{B}_{22}(t)}{\left(T_{0}-t\right)^{2}}-\frac{q_{4} q_{9}(t) \bar{L}_{22}(t)}{\left(q_{1} q_{2}-q_{3}^{2}\right) \gamma} \\
& \begin{array}{l}
+\frac{q_{4} q_{8} A_{22}(t)}{\left(q_{1} q_{2}-q_{3}^{2}\right) \gamma \bar{A}_{22}^{2}(t)}+\frac{\delta^{2} A_{22}(t) \bar{A}_{22}(t)}{\gamma \zeta^{2} h^{P} \bar{A}_{1}(t)}-\frac{\delta \bar{A}_{22}(t)}{\gamma \zeta \bar{A}_{1}(t)}+\frac{\delta \bar{A}_{22}(t)}{\zeta \bar{A}_{1}(t)} \bar{N}_{1}(t)-\frac{\delta A_{22}(t)}{\gamma \zeta \bar{A}_{1}(t)}+\frac{h^{P}}{\gamma}=0, \\
\bar{N}_{22}(t)=0 .
\end{array}
\end{aligned}
$$


By solving the above equations ((72) and (73)), we have

$$
\begin{aligned}
& \left\{\begin{array}{l}
A_{22}(t)=\bar{A}_{22}(t)=e^{r(T-t)}, \\
\bar{B}_{22}(t)=e^{-(\delta / \zeta)(T-t)}\left\{\int_{s}^{T} \frac{\delta \bar{B}_{1}(s) e^{(\delta / \zeta)(T-s)}}{\zeta} \mathrm{d} s\right\}, \\
\bar{L}_{22}(t)=e^{-\frac{\delta(T-t)}{\zeta}}\left\{\int_{s}^{T}\left[-\frac{q_{4} \sigma_{1} q_{3}}{\left(q_{1} q_{2}-q_{3}^{2}\right) \gamma e^{r(T-s)}}-\frac{2 q_{4} q_{9}(s) \bar{B}_{22}(s)}{\left(q_{1} q_{2}-q_{3}^{2}\right) \gamma}+\frac{\delta \bar{L}_{1}(s)}{\zeta}\right] e^{(\delta(T-s) / \zeta)} \mathrm{d} s\right\} \\
\bar{N}_{22}(t)=e^{-(\delta / \zeta)(T-t)}\left\{\int _ { s } ^ { T } \left[\left(c-c_{1}\right) e^{r(T-s)}+\frac{\bar{B}_{22}(s)}{\left(T_{0}-s\right)^{2}}-\frac{q_{4} q_{9}(s) \bar{L}_{22}(s)}{\left(q_{1} q_{2}-q_{3}^{2}\right) \gamma}\right.\right. \\
\left.\left.+\frac{q_{4} q_{8}}{\left(q_{1} q_{2}-q_{3}^{2}\right) \gamma}+\frac{\delta^{2} e^{r(T-s)}}{\gamma \zeta^{2} h^{P}}-\frac{\delta}{\gamma \zeta}+\frac{\delta}{\zeta} \bar{N}_{1}(t)-\frac{\delta}{\gamma \zeta}+\frac{h^{P}}{\gamma}\right] e^{-(\delta / \zeta)(T-s)} \mathrm{d} s\right\}
\end{array}\right. \\
& \int B_{22}(t)=e^{-h^{P}(T-t)}\left\{\int _ { s } ^ { T } \left\{-\frac{2 \gamma \bar{B}_{22}^{2}(s)}{\left(T_{0}-s\right)^{2}}-\frac{\gamma q_{2}}{2} \bar{\Gamma}_{42}^{2}(s) e^{2 r(T-s)}+\bar{\Gamma}_{42}(s) \bar{\Gamma}_{62}(s)\right.\right. \\
& \left.\left.+\frac{\delta}{\zeta}\left[\bar{B}_{1}(s)-\bar{B}_{22}(s)\right]+h^{P}\left[B_{1}(s)-\bar{B}_{1}(s)+\bar{B}_{22}(s)\right]\right\} e^{h^{P}(T-s)} \mathrm{d} s\right\} \\
& L_{22}(t)=e^{-h^{P}(T-t)}\left\{\int _ { s } ^ { T } \left\{-\frac{2 \gamma \bar{B}_{22}(s) \bar{L}_{22}(s)}{\left(T_{0}-s\right)^{2}}-\frac{\gamma q_{2}}{2} \bar{\Gamma}_{32}(s) \bar{\Gamma}_{42}(s) e^{2 r(T-s)}-\bar{\Gamma}_{32}(s) \bar{\Gamma}_{62}(s)\right.\right. \\
& \left.\left.\left\{-\bar{\Gamma}_{42}(s) \bar{\Gamma}_{52}(s)\right)+\frac{\delta}{\zeta}\left[\bar{L}_{1}(s)-\bar{L}_{2}(s)\right]+h^{P}\left[L_{1}(s)-\bar{L}_{1}(s)+\bar{L}_{22}(s)\right]\right\} e^{h^{P}(T-s)} \mathrm{d} s\right\}, \\
& N_{22}(t)=e^{-h^{P}(T-t)}\left\{\int _ { s } ^ { T } \left\{\left(c-c_{1}\right) e^{r(T-s)}+\frac{B_{22}(s)}{\left(T_{0}-s\right)^{2}}-\frac{\gamma \bar{L}_{1}^{2}(s)}{2\left(T_{0}-s\right)^{2}}\right.\right. \\
& -\frac{\gamma q_{2}}{2} e^{2 r(T-s)} \bar{\Gamma}_{32}^{2}(s)+\bar{\Gamma}_{32}(s) \bar{\Gamma}_{52}(s)+\frac{\delta^{2}}{\gamma \zeta^{2} h^{P}}-\frac{2 \delta}{\gamma \zeta}+\frac{\delta}{\zeta}\left[\bar{N}_{1}(s)-\bar{N}_{22}(s)\right] \\
& \left.\left.+h^{P}\left[N_{1}(s)-\bar{N}_{1}(s)+\bar{N}_{22}(s)\right]-\frac{h^{P}}{\gamma}-\frac{\gamma\left(h^{P}\right)^{2}}{2}\left[\frac{1}{\gamma}-\frac{\delta}{\gamma \zeta h^{P}}\right]^{2}\right\} e^{h^{P}(T-s)} \mathrm{d} s\right\} .
\end{aligned}
$$

In summary of the above derivation and according to Theorem 2, we derive the following theorem.

Theorem 5. For the predefault case, i.e., the case $z=0$, the solution to problem (16) is given as follows. The closed-loop equilibrium reinsurance strategy is given by

$$
a^{*}(t)=\frac{q_{6}-q_{7}(t) \bar{L}_{21}(t)-\left[\sigma_{1} q_{2}+2 q_{7}(t) \bar{B}_{21}(t)\right] m}{\left(q_{1} q_{2}-q_{3}^{2}\right) \gamma e^{r(T-t)}} \vee 0
$$

the closed-loop equilibrium investment strategies are given by

$$
\pi_{1}^{*}(t)= \begin{cases}\frac{q_{8}-q_{9}(t) \bar{L}_{21}(t)-\left[\sigma_{1} q_{3}+2 q_{9}(t) \bar{B}_{21}(t)\right] m}{\left(q_{1} q_{2}-q_{3}^{2}\right) \gamma e^{r(T-t)}}, & \text { for } t \in \mathcal{O}_{3}, \\ \frac{q_{8}-q_{9}(t) \bar{L}_{22}(t)-\left[\sigma_{1} q_{3}+2 q_{9}(t) \bar{B}_{22}(t)\right] m}{\left(q_{1} q_{2}-q_{3}^{2}\right) \gamma e^{r(T-t)}}, & \text { for } t \in \mathcal{O}_{4},\end{cases}
$$

and 


$$
\pi_{2}^{*}(t)=\left\{\begin{array}{l}
\text { for } t \in \mathcal{O}_{1} \cap \mathcal{O}_{3}: \\
\frac{1}{\zeta e^{r(T-t)}}\left\{\frac{\delta}{\gamma \zeta h^{P}}-\frac{1}{\gamma}+\left[\bar{B}_{11}(t)-\bar{B}_{21}(t)\right] m^{2}+\left[\bar{L}_{11}(t)-\bar{L}_{21}(t)\right] m+\bar{N}_{11}(t)-\bar{N}_{21}(t)\right\}, \\
\text { for } t \in \mathcal{O}_{1} \cap \mathcal{O}_{4}: \\
\frac{1}{\zeta e^{r(T-t)}}\left\{\frac{\delta}{\gamma \zeta h^{P}}-\frac{1}{\gamma}+\left[\bar{B}_{11}(t)-\bar{B}_{22}(t)\right] m^{2}+\left[\bar{L}_{11}(t)-\bar{L}_{22}(t)\right] m+\bar{N}_{11}(t)-\bar{N}_{22}(t)\right\}, \\
\text { for } t \in \mathcal{O}_{2} \cap \mathcal{O}_{3}: \\
\frac{1}{\zeta e^{r(T-t)}}\left\{\frac{\delta}{\gamma \zeta h^{P}}-\frac{1}{\gamma}+\left[\bar{B}_{12}(t)-\bar{B}_{21}(t)\right] m^{2}+\left[\bar{L}_{12}(t)-\bar{L}_{21}(t)\right] m+\bar{N}_{12}(t)-\bar{N}_{21}(t)\right\}, \\
\text { for } t \in \mathcal{O}_{2} \cap \mathcal{O}_{4}: \\
\frac{1}{\zeta e^{r(T-t)}}\left\{\frac{\delta}{\gamma \zeta h^{P}}-\frac{1}{\gamma}+\left[\bar{B}_{12}(t)-\bar{B}_{22}(t)\right] m^{2}+\left[\bar{L}_{12}(t)-\bar{L}_{22}(t)\right] m+\bar{N}_{12}(t)-\bar{N}_{22}(t)\right\}
\end{array}\right.
$$

the corresponding value function is given by

$$
W(t, x, m, 0)= \begin{cases}x e^{r(T-t)}+B_{21}(t) m^{2}+L_{21}(t) m+N_{21}(t), & \text { for } t \in \mathcal{O}_{3}, \\ x e^{r(T-t)}+B_{22}(t) m^{2}+L_{22}(t) m+N_{22}(t), & \text { for } t \in \mathcal{O}_{4},\end{cases}
$$

and the corresponding variance of the terminal wealth is given

by

$$
\operatorname{Var}_{t, x, m, 0}\left(X_{T}^{\pi^{*}}\right)= \begin{cases}\frac{2}{\gamma}\left\{\left[B_{21}-\bar{B}_{21}\right] m^{2}+\left[L_{21}-\bar{L}_{21}\right] m+N_{21}-\bar{N}_{21}\right\}, & \text { for } t \in \mathcal{O}_{3}, \\ \frac{2}{\gamma}\left\{\left[B_{22}-\bar{B}_{22}\right] m^{2}+\left[L_{22}-\bar{L}_{22}\right] m+N_{22}-\bar{N}_{22}\right\}, & \text { for } t \in \mathcal{O}_{4} .\end{cases}
$$

\section{Numerical Analysis}

In this section, we conduct a series of numerical examples to illustrate the effects of some important model parameters on the closed-loop equilibrium reinsurance and investment strategies for the case of predefault. Since we consider insider information in the insurance market, we also analyze the similarities and differences between the closed-loop equilibrium reinsurance strategy with and without insider information. Throughout the numerical analysis, unless otherwise stated, $t \in \mathcal{O}_{1} \cap \mathcal{O}_{3}$ and the basic parameters are given by $\sigma_{1}=0.2, \lambda_{1}=1, \lambda_{2}=2, \lambda=2, \sigma_{2}=0.5, \rho=0.1$, $\mu=0.08, r=0.02, c_{1}=1, \delta_{0}=1, \gamma=1, \delta=0.01, \zeta=0.6$, $T=10, t=5, T_{0}=12$, and $m=3$.
Suppose that both the size $Y_{i}$ of claim and the size $U_{i}$ of price jump follow exponential distributions with paraments 1 and 1, respectively, i.e., the density functions of $Y_{i}$ and $U_{i}$ are given by $f_{Y}(y)=e^{-y}, y \geq 0$, and $f_{U}(u)=e^{-u}, u \geq 0$. Hence, we can obtain $\mu_{11}=\mu_{21}=1$ and $\mu_{12}=\mu_{22}=2$.

5.1. Closed-Loop Equilibrium Reinsurance Strategy $a^{*}(t)$. In this section, we present the numerical results for the closed-loop equilibrium reinsurance strategy $a^{*}(t)$, which is determined by (75).

Example 1. Assume that $\sigma_{1} \in[0.1,0.26]$, and Figure 1 shows the influence of $\sigma_{1}$ on $a^{*}(t)$ when not considering the insider 


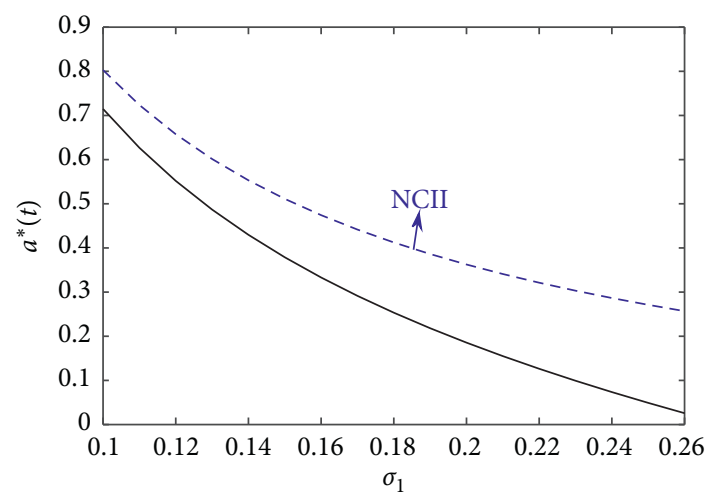

Figure 1: Effect of $\sigma_{1}$ on $a^{*}(t)$.

information (the curves marked with NCII) and considering the insider information (the curves not marked), respectively. From Figure 1, we see that whether the insider information is considered or not, $a^{*}(t)$ is a decreasing function of $\sigma_{1}, \sigma_{1}$ is associated with the uncertainty of the claim, and the larger $\sigma_{1}$ is, the greater the claim risk will be. This will enhance the insurer's reinsurance willingness. Therefore, from Figure 1, we can see whether the insider information is considered or not and $a^{*}(t)$ decreases with respect to $\sigma_{1}$. From Figure 1, we also find that when considering the insider information, the retention level changes more significantly. This may be because the insurer will adjust the reinsurance decision in time according to the insider information he knows.

Example 2. Assume that $\lambda_{1} \in[0.8,1.5]$, and Figure 2 displays the effect of $\lambda_{1}$ on $a^{*}(t)$ with and without considering the insider information, respectively. A bigger $\lambda_{1}$ implies that the jump intensity in the surplus process becomes larger, which will result in more claim risks. Naturally, the insurer will reduce his retention and transfer more claim risks to the reinsurer. Therefore, from Figure 2, we find that whether the insider information is considered or not, $a^{*}(t)$ is a decreasing function of $\lambda_{1}$.

Example 3. Assume that $\rho \in[0.1,0.6]$, and Figure 3 illustrates the influence of $\rho$ on $a^{*}(t)$ with and without considering the insider information, respectively. $\rho$ reflects the correlation coefficient between the uncertainty of claims and the price volatility. The larger $\rho$ is, the more claim and price risk will be. This will enhance the insurer's reinsurance willingness. Therefore, from Figure 3, we can see whether the insider information is considered or not and $a^{*}(t)$ decreases with respect to $\rho$.

Further examining Figures 1-3, we have the following findings:

(i) Whether the insider information is considered or not, the trends of the closed-loop equilibrium reinsurance strategy with respect to relevant model parameters are consistent, but there are significant differences in the size of the retention level.

(ii) Compared with the case considering the insider information, the insurer keeps a larger retention

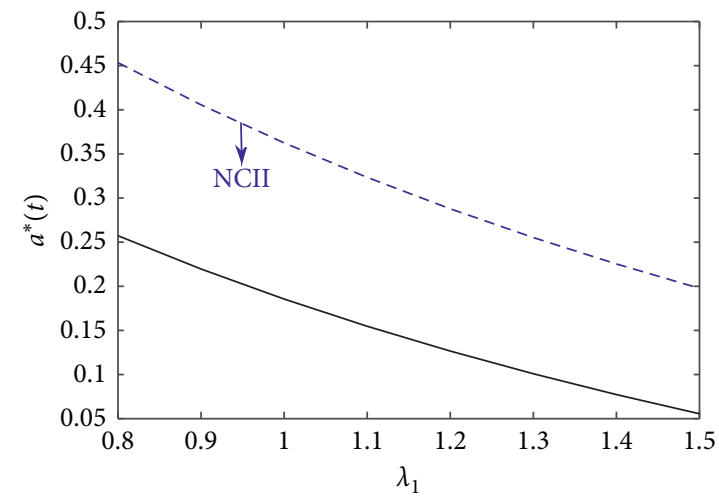

Figure 2: Effect of $\lambda_{1}$ on $a^{*}(t)$.

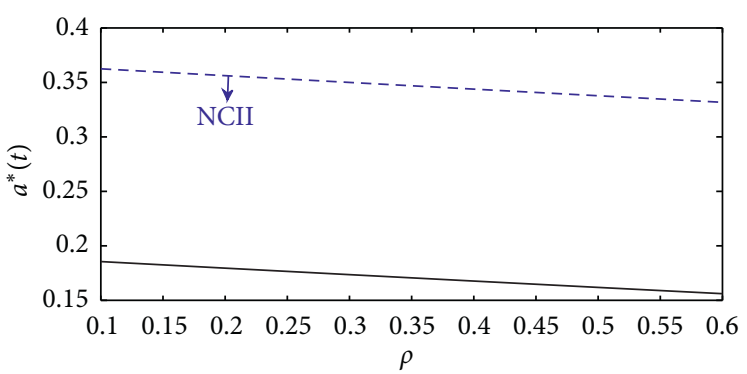

FIgURE 3: Effect of $\rho$ on $a^{*}(t)$.

level when the insider information is not considered. The reason may be that the insurer failed to obtain the insider information of claims and could not adjust the reinsurance decision in time.

From these findings, we can draw the conclusion that the insider information of claims has an important influence on the insurer's reinsurance decision, and it can enable the insurer to effectively avoid claims risks through reinsurance.

5.2. Closed-Loop Equilibrium Investment Strategy $\pi_{1}^{*}(t)$. In this section, we present the numerical results for the closed-loop equilibrium investment strategy $\pi_{1}^{*}(t)$, which is determined by (76).

Example 4. Assume that $\mu \in[0.05,0.09]$, and Figure 4 shows the effect of $\mu$ on $\pi_{1}^{*}(t)$. From Figure 4 , it is evident that $\pi_{1}^{*}(t)$ is an increasing function of $\mu$. $\mu$ describes the expected income of the risky asset. The larger $\mu$ is, the greater the expected income of the risky asset will be, and hence, the more the insurer will wish to invest in the risky asset.

Example 5. Assume that $\sigma_{2} \in[0.2,0.6]$, and Figure 5 displays the effect of $\sigma_{2}$ on $\pi_{1}^{*}(t)$. From Figure 5 , it can be seen that $\pi_{1}^{*}(t)$ is a decreasing function of $\sigma_{2}$, which is the volatility of the risky asset. The larger $\sigma_{2}$ is, the riskier the risky asset will be, and hence, the less the insurer will wish to invest in the risky asset.

Example 6. Assume that $\lambda_{2} \in[1,5]$, and Figure 6 illustrates the effect of $\lambda_{2}$ on $\pi_{1}^{*}(t)$. From Figure 6 , we can see that $\pi_{1}^{*}(t)$ 


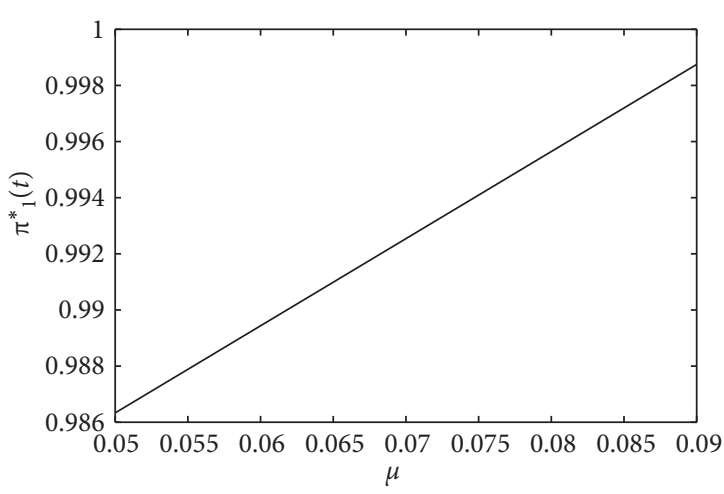

FIgURE 4: Effect of $\mu$ on $\pi_{1}^{*}(t)$.

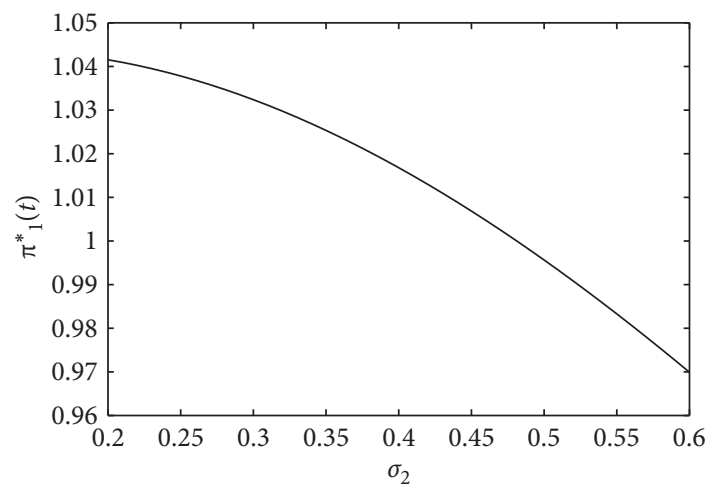

Figure 5: Effect of $\sigma_{2}$ on $\pi_{1}^{*}(t)$.

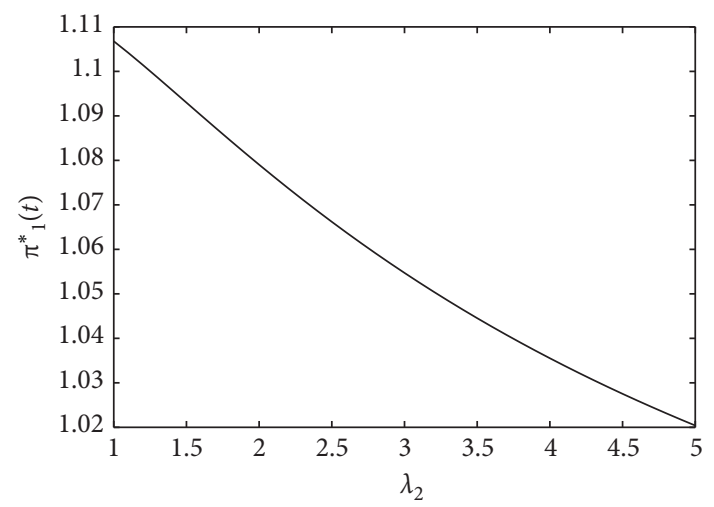

Figure 6: Effect of $\lambda_{2}$ on $\pi_{1}^{*}(t)$.

is a decreasing function of $\lambda_{2}$. $\lambda_{2}$ represents the jump intensity of the risky asset, and the larger $\lambda_{2}$ is, the greater the risk will be; therefore, the insurer reduces his investment in the risky asset.

Example 7. Assume that $\rho \in[0.1,0.6]$, and Figure 7 shows the effect of $\rho$ on $\pi_{1}^{*}(t)$. From Figure 7, it can be seen that $\pi_{1}^{*}(t)$ is an increasing function of $\rho$. When the correlation between the insurance market and the financial market increases, the insurer will increase his investment in the risky asset. From Example 3 and Example 7, we find that the effect of $\rho$ on the closed-loop equilibrium investment strategy and reinsurance strategy is inconsistent.

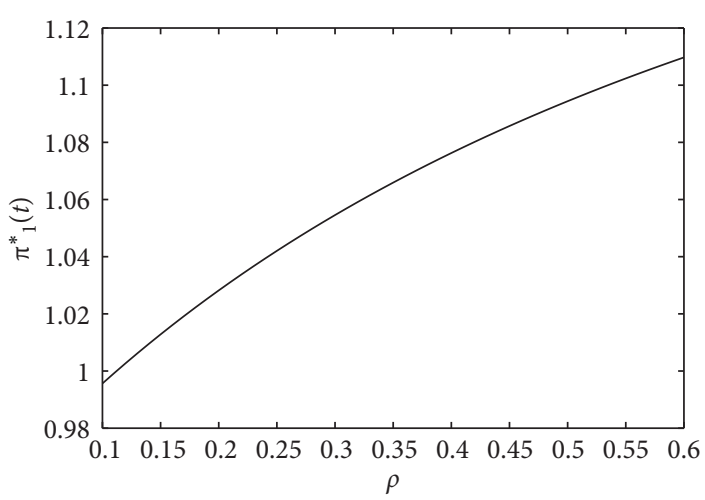

Figure 7: Effect of $\rho$ on $\pi_{1}^{*}(t)$.

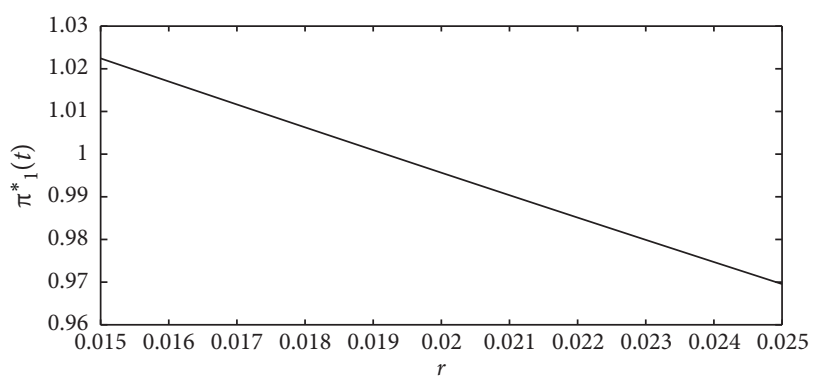

FiguRE 8: Effect of $r$ on $\pi_{1}^{*}(t)$.

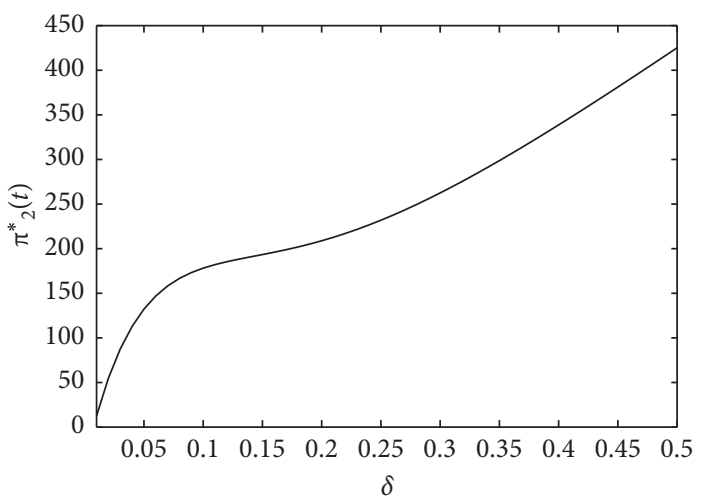

Figure 9: Effect of $\delta$ on $\pi_{2}^{*}(t)$.

Example 8. Assume that $r \in[0.015,0.025]$, and Figure 8 shows the effect of $r$ on $\pi_{1}^{*}(t)$. From Figure 8 , it is evident that $\pi_{1}^{*}(t)$ is a decreasing function of $r . r$ is the interest rate of the risk-free. The larger $r$ is, the greater the expected income of the risk-free asset will be, and hence, the more the insurer will wish to invest in the risk-free asset.

5.3. Closed-Loop Equilibrium Investment Strategy $\pi_{2}^{*}(t)$. In this section, we present the numerical results for the closed-loop equilibrium investment strategy $\pi_{2}^{*}(t)$, which is determined by (77).

Example 9. Assume that $\delta \in[0.01,0.5]$ and $m=1$, and Figure 9 shows the effect of $\delta$ on $\pi_{2}^{*}(t)$. Figure 9 implies that 


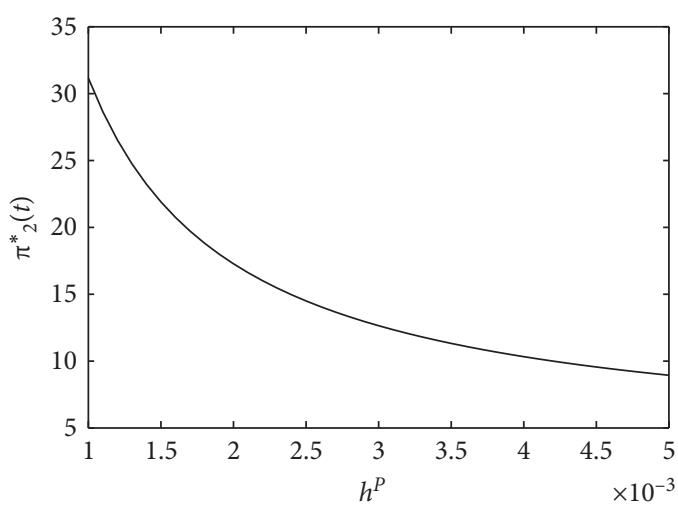

Figure 10: Effect of $h^{P}$ on $\pi_{2}^{*}(t)$.

the insurer will invest more in the defaultable bond when the credit spread $\delta$ is higher. It is intuitive, as the larger the value of $\delta$ is, the defaultable bond will be more attractive.

Example 10. Assume that $h^{P} \in[0.001,0.005]$ and $m=1$, and Figure 10 illustrates the effect of $h^{P}$ on $\pi_{2}^{*}(t)$. From Figure 10, we can see that $\pi_{2}^{*}(t)$ is a decreasing function of $h^{P}$. As $h^{P}$ represents the intensity of the defaultable, the larger $h^{P}$ is, the less attractive the defaultable bond will be, and hence, the less the insurer will invest in the defaultable bond.

\section{Conclusions}

We have investigated the closed-loop equilibrium investment and reinsurance problem with insider information and default risk. The insurer can purchase reinsurance or acquire new business to transfer potential claim risk and invest in one riskfree asset, one risky asset, and one defaultable bond to increase wealth, where the price process of the risky asset is modulated by a jump-diffusion process. Compared with current literature studies, such as Cao et al. [20], Zhao et al. [22], and Zhang and Qiao [23], there are two innovations in this paper. First, the insider information and default risk are simultaneously considered. Second, two kinds of dependencies between the insurance market and the financial market are considered. By adopting the game theoretic framework, we derived the closedloop equilibrium investment and reinsurance strategies which are time-consistent. We systematically examine the influences of the insider information and some important model parameters on reinsurance and investment strategies through numerical experiments and derive some interesting observations. It is worth noting that it is also interesting to consider the open-loop equilibrium strategy. We would like to leave it as a further research in the future.

\section{Data Availability}

The data used to support the findings of this study are available from the corresponding author upon request.

\section{Conflicts of Interest}

The author declares that there are no conflicts of interest regarding the publication of this paper.

\section{Acknowledgments}

This research was supported by the Natural Science Basic Research Program of Shaanxi grant no. 2020JM-646.

\section{References}

[1] H. Markowitz, "Portfolio selection," The Journal of Finance, vol. 7, no. 1, pp. 77-91, 1952.

[2] X. Y. Zhou and D. Li, "Continuous-time mean-variance portfolio selection: a stochastic LQ framework," Applied Mathematics and Optimization, vol. 42, no. 1, pp. 19-33, 2000.

[3] N. Bäuerle, "Benchmark and mean-variance problems for insurers," Mathematical Methods of Operations Research, vol. 62, no. 1, pp. 159-165, 2005.

[4] Z. Liang, J. Bi, K. C. Yuen, and C. Zhang, "Optimal meanvariance reinsurance and investment in a jump-diffusion financial market with common shock dependence," Mathematical Methods of Operations Research, vol. 84, no. 1, pp. 155-181, 2016.

[5] R. H. Strotz, "Myopia and inconsistency in dynamic utility maximization," The Review of Economic Studies, vol. 23, no. 3, pp. 165-180, 1955.

[6] I. Ekeland and A. Lazrak, "Being serious about non-commitment: subgame perfect equilibrium in continuous time," 2006, http://arxiv.org/abs/0604264.

[7] Bj, T. rk and A. Murgoci, "A general theory of Markovian time inconsistent stochastic control problems," 2010, http://ssrn. com/abstract $=1694759$.

[8] X. Lin and Y. Qian, "Time-consistent mean-variance reinsurance-investment strategy for insurers under CEV model," Scandinavian Actuarial Journal, vol. 2016, no. 7, 671 pages, Article ID 1048710, 2016.

[9] C. Zhang and Z. Liang, "Portfolio optimization for jumpdiffusion risky assets with common shock dependence and state dependent risk aversion," Optimal Control Applications and Methods, vol. 38, no. 2, pp. 229-246, 2017.

[10] D. Hu and H. Wang, "Time-consistent investment and reinsurance under relative performance concerns," Communications in Statistics-Theory and Methods, vol. 47, no. 7, pp. 1693-1717, 2018.

[11] P. Yang, Z. Chen, and L. Wang, "Time-consistent reinsurance and investment strategy combining quota-share and excess of loss for meanvariance insurers with jump-diffusion price process," Communications in Statistics-Theory and Methods, 2019.

[12] P. Yang, Z. Chen, and Y. Xu, "Time-consistent equilibrium reinsurance-investment strategy for $\mathrm{n}$ competitive insurers under a new interaction mechanism and a general investment framework," Journal of Computational And Applied Mathematics, vol. 374, Article ID 112769, 2020.

[13] Y. Hu, H. Jin, and X. Y. Zhou, "Time-inconsistent stochastic linear--quadratic control," SIAM Journal on Control and Optimization, vol. 50, no. 3, pp. 1548-1572, 2012.

[14] Y. Hu, H. Jin, and X. Y. Zhou, “Time-inconsistent stochastic linear-quadratic control: characterization and uniqueness of equilibrium," SIAM Journal on Control and Optimization, vol. 55, no. 2, pp. 261-1279, 2017.

[15] Z. Sun and X. Guo, "Equilibrium for a time-inconsistent stochastic linear-quadratic control system with jumps and its application to the mean-variance problem," Journal of $O p$ timization Theory and Applications, vol. 181, no. 2, pp. 83-410, 2019. 
[16] T. Yan and H. Y. Wong, "Open-loop equilibrium strategy for mean-variance portfolio problem under stochastic volatility," Automatica, vol. 107, pp. 211-223, 2019.

[17] T. Yan and H. Y. Wong, "Open-loop equilibrium reinsuranceinvestment strategy under mean-variance criterion with stochastic volatility," Insurance: Mathematics and Economics, vol. 90, pp. 105-119, 2020.

[18] T. Wang, "Uniqueness of equilibrium strategies in dynamic mean-variance problems with random coefficients," Journal of Mathematical Analysis and Applications, vol. 490, no. 1, p. 124199, 2020.

[19] I. D. Baltas, N. E. Frangos, and A. N. Yannacopoulos, "Optimal investment and reinsurance policies in insurance markets under the effect of inside information," Applied Stochastic Models in Business and Industry, vol. 28, no. 6, pp. 506-528, 2012.

[20] J. Cao, X.-C. Peng, and Y.-J. Hu, "Optimal time-consistent investment and reinsurance strategy for mean-variance insurers under the inside information," Acta Mathematicae Applicatae Sinica, English Series, vol. 32, no. 4, pp. 1087-1100, 2016.

[21] J. Xiong, S. Zhang, H. Zhao, and X. Zeng, "Optimal proportional reinsurance and investment problem with jumpdiffusion risk process under effect of inside information," Frontiers of Mathematics in China, vol. 9, no. 4, pp. 965-982, 2014.

[22] H. Zhao, Y. Shen, and Y. Zeng, "Time-consistent investmentreinsurance strategy for mean-variance insurers with a defaultable security," Journal of Mathematical Analysis and Applications, vol. 437, no. 2, pp. 1036-1057, 2016.

[23] Q. Zhang and P. Chen, "Time-consistent mean-variance proportional reinsurance and investment problem in a defaultable market," Optimization, vol. 67, no. 5, pp. 683-699, 2018.

[24] J. Zhu, G. Guan, and S. Li, "Time-consistent non-zero-sum stochastic differential reinsurance and investment game under default and volatility risks," Journal of Computational and Applied Mathematics, vol. 374, p. 112737, 2020.

[25] P. Protter, Stochastic Integration and Stochastic Differential Equations, Springer, Berlin, Germany, 2004.

[26] T. R. Bielecki and I. Jang, "Portfolio optimization with a defaultable security," Asia-Pac Financial Markets, vol. 13, no. 2, pp. 113-127, 2006.

[27] C. Czichowsky, "Time-consistent mean-variance portfolio selection in discrete and continuous time," Finance and Stochastics, vol. 17, no. 2, pp. 227-271, 2013. 